\title{
ON THE STRUCTURE OF ASYMPTOTIC $\ell_{p}$ SPACES
}

\author{
E. ODELL, TH. SCHLUMPRECHT AND A. ZSÁK
}

\begin{abstract}
We prove that if $X$ is a separable, reflexive space which is asymptotic $\ell_{p}$ for some $1 \leq p \leq \infty$, then $X$ embeds into a reflexive space $Z$ having an asymptotic $\ell_{p}$ finitedimensional decomposition. This result leads to an intrinsic characterization of subspaces of spaces with an asymptotic $\ell_{p}$ FDD. More general results of this type are also obtained.
\end{abstract}

\section{INTRODUCTION}

Let $X$ be a separable Banach space with a finite-dimensional decomposition (FDD), $\left(E_{n}\right)$. Let $1 \leq p \leq \infty$. $X$ is asymptotic $\ell_{p}$ with respect to $\left(E_{n}\right)[\mathrm{MT}$ if there exists $C<\infty$ so that for all $n$ and all block sequences $\left(x_{i}\right)_{i=1}^{n}$ of $\left(E_{i}\right)_{i=n}^{\infty}$

$$
\frac{1}{C}\left(\sum_{i=1}^{n}\left\|x_{i}\right\|^{p}\right)^{1 / p} \leq\left\|\sum_{i=1}^{n} x_{i}\right\| \leq C\left(\sum_{i=1}^{n}\left\|x_{i}\right\|^{p}\right)^{1 / p}
$$

(if $p=\infty$ we use the $c_{0}$-norm $\max \left\|x_{i}\right\|$ ).

A coordinate-free version of this notion is as follows [MMT]. Let $X$ be an arbitrary Banach space, and let $\operatorname{cof}(X)$ denote the set of all closed subspaces of $X$ having finite codimension. We say $X$ is asymptotic $\ell_{p}$ if there exists $C<\infty$ so that

$$
\begin{aligned}
\forall n \in \mathbb{N} \quad & \exists Y_{1} \in \operatorname{cof}(X) \forall y_{1} \in S_{Y_{1}} \text { (unit sphere of } Y_{1} \text { ) } \\
& \exists Y_{2} \in \operatorname{cof}(X) \forall y_{2} \in S_{Y_{2}} \\
& \vdots \\
& \exists Y_{n} \in \operatorname{cof}(X) \forall y_{n} \in S_{Y_{n}} \\
& \left(y_{i}\right)_{i=1}^{n} \text { is } C \text {-equivalent to the unit vector basis of } \ell_{p}^{n} .
\end{aligned}
$$

We prove (Corollary 4.9) that if $X$ is separable, reflexive and asymptotic $\ell_{p}$, then there exists a reflexive space $Z$ with an $\operatorname{FDD}\left(E_{n}\right)$ so that $Z$ is asymptotic $\ell_{p}$ with respect to $\left(E_{n}\right)$ and $X$ embeds isomorphically into $Z$. This is deduced from a more general result (Corollary 4.8) which considers separable, reflexive spaces $X$ that satisfy the following for some $1 \leq q \leq p \leq \infty$ and $C<\infty$.

$$
\begin{aligned}
\forall n \in \mathbb{N} & \exists Y_{1} \in \operatorname{cof}(X) \forall y_{1} \in S_{Y_{1}} \\
& \exists Y_{2} \in \operatorname{cof}(X) \forall y_{2} \in S_{Y_{2}} \\
& \vdots \\
& \exists Y_{n} \in \operatorname{cof}(X) \forall y_{n} \in S_{Y_{n}}
\end{aligned}
$$

Date: $20 / 2 / 2006$.

2000 Mathematics Subject Classification. Primary 46B20; Secondary 46B03, 46B10.

Research of the first two authors was supported by the National Science Foundation. 


$$
\frac{1}{C}\left(\sum_{i=1}^{n}\left|a_{i}\right|^{p}\right)^{1 / p} \leq\left\|\sum_{i=1}^{n} a_{i} y_{i}\right\| \leq C\left(\sum_{i=1}^{n}\left|a_{i}\right|^{q}\right)^{1 / q} \text { whenever }\left(a_{i}\right)_{i=1}^{n} \subset \mathbb{R} .
$$

We characterize such spaces as those that embed into reflexive spaces $Z$ with an FDD $\left(E_{i}\right)$ satisfying asymptotic $\left(\ell_{p}, \ell_{q}\right)$-estimates. This means that for some $C<\infty$, for all $n \in \mathbb{N}$ and all block sequences $\left(x_{i}\right)_{i=1}^{n}$ of $\left(E_{i}\right)_{i=n}^{\infty}$

$$
\frac{1}{C}\left(\sum_{i=1}^{n}\left\|x_{i}\right\|^{p}\right)^{1 / p} \leq\left\|\sum_{i=1}^{n} x_{i}\right\| \leq C\left(\sum_{i=1}^{n}\left\|x_{i}\right\|^{q}\right)^{1 / q} .
$$

We also show that this is equivalent to $X$ being a quotient of such a space $Z$.

To accomplish that we develop a more general machinery concerning asymptotic $U$ upper and $V$-lower estimates, where $U$ and $V$ are certain spaces with subsymmetric bases. Theorem 4.7 is concerned with this general setting, and Corollary 4.8 will then be obtained as a special case.

In an earlier paper OS1 analogous results were obtained characterizing when a reflexive space embeds into $\left(\oplus_{i=1}^{\infty} E_{i}\right)_{\ell_{p}}$, the $\ell_{p}$-sum of some sequence $E=\left(E_{i}\right)$ of finite-dimensional spaces. In this paper the role of $\left(\oplus_{i=1}^{\infty} E_{i}\right)_{\ell_{p}}$ is played by a space $Z_{V}(E)$, where $V$ is a space with an unconditional basis, especially a convexified Tsirelson space. Section 2 contains the precise definition of $Z_{V}(E)$ and some structural results about it. In section 3 we present several embedding theorems (Theorem 3.1 Corollary 3.2 and Theorem 3.4) which characterize when a reflexive space embeds into some $Z_{V}(E)$ and is a quotient of some $Y_{V}(F)$. Section 4 contains our main results.

Before proceeding we first introduce some definitions and notation.

Let $Z$ be a Banach space with an FDD $E=\left(E_{n}\right)$. For $n \in \mathbb{N}$ we denote the $n$-th coordinate projection by $P_{n}^{E}$, i.e. $P_{n}^{E}: Z \rightarrow E_{n}, \quad \sum z_{i} \mapsto z_{n}$. For finite $A \subset \mathbb{N}$ we put $P_{A}^{E}=\sum_{n \in A} P_{n}^{E}$. The projection constant of $\left(E_{n}\right)$ (in $Z$ ) is defined by

$$
K=K(E, Z)=\sup _{m \leq n}\left\|P_{[m, n]}^{E}\right\| \text {. }
$$

Recall that $K$ is always finite and, as in the case of bases, we call $\left(E_{n}\right)$ bimonotone (in $Z$ ) if $K=1$. By passing to the equivalent norm

$$
\|\cdot\|: Z \rightarrow \mathbb{R}, \quad z \mapsto \sup _{m \leq n}\left\|P_{[m, n]}^{E}(z)\right\|,
$$

we can always renorm $Z$ so that $K=1$.

For a sequence $\left(E_{i}\right)$ of finite-dimensional spaces we define the vector space

$$
\mathrm{c}_{00}\left(\oplus_{i=1}^{\infty} E_{i}\right)=\left\{\left(z_{i}\right): z_{i} \in E_{i} \text { for } i \in \mathbb{N}, \text { and }\left\{i \in \mathbb{N}: z_{i} \neq 0\right\} \text { is finite }\right\},
$$

which is dense in each Banach space for which $\left(E_{i}\right)$ is an FDD. For $A \subset \mathbb{N}$ we denote by $\oplus_{i \in A} E_{i}$ the linear subspace of $c_{00}\left(\oplus E_{i}\right)$ generated by the elements of $\bigcup_{i \in A} E_{i}$. As usual we denote the vector space of sequences in $\mathbb{R}$ which are eventually zero by $c_{00}$ and its unit vector basis by $\left(e_{i}\right)$. We sometimes will consider for the same sequence $\left(E_{i}\right)$ of finite-dimensional spaces different norms on $c_{00}\left(\oplus E_{i}\right)$. In order to avoid confusion we will therefore often index the norm by the Banach space whose norm we are using, i.e. $\|\cdot\|_{Z}$ denotes the norm of the Banach space $Z$.

If $Z$ has an FDD $\left(E_{i}\right)$, the vector space $c_{00}\left(\oplus_{i=1}^{\infty} E_{i}^{*}\right)$, where $E_{i}^{*}$ is the dual space of $E_{i}$ for $i \in \mathbb{N}$, is a $w^{*}$-dense subspace of $Z^{*}$. We denote the norm closure of $c_{00}\left(\oplus_{i=1}^{\infty} E_{i}^{*}\right)$ in $Z^{*}$ by $Z^{(*)}$. $Z^{(*)}$ is $w^{*}$-dense in $Z^{*}$, the unit ball $B_{Z^{(*)}}$ norms $Z$, and $\left(E_{i}^{*}\right)$ is an FDD of 
$Z^{(*)}$ having a projection constant not exceeding $K(E, Z)$. If $K(E, Z)=1$ then $B_{Z^{(*)}}$ is 1-norming and $Z^{(*)(*)}=Z$.

For $z \in \mathrm{c}_{00}\left(\oplus E_{i}\right)$ we define the E-support of $z$ by

$$
\operatorname{supp}_{E}(z)=\left\{i \in \mathbb{N}: P_{i}^{E}(z) \neq 0\right\} .
$$

A sequence $\left(z_{j}\right)$ (finite or infinite) of non-zero vectors in $c_{00}\left(\oplus E_{i}\right)$ is called a block sequence of $\left(E_{i}\right)$ if

$$
\max \operatorname{supp}_{E}\left(z_{n}\right)<\min \operatorname{supp}_{E}\left(z_{n+1}\right) \quad \text { whenever } n \in \mathbb{N}\left(\text { or } n<\operatorname{length}\left(z_{j}\right)\right),
$$

and it is called a skipped block sequence of $\left(E_{i}\right)$ if

$$
\max \operatorname{supp}_{E}\left(z_{n}\right)<\min \operatorname{supp}_{E}\left(z_{n+1}\right)-1 \quad \text { whenever } n \in \mathbb{N}\left(\text { or } n<\operatorname{length}\left(z_{j}\right)\right) \text {. }
$$

Let $\bar{\delta}=\left(\delta_{n}\right) \subset(0,1)$ with $\delta_{n} \downarrow 0$. A (finite or infinite) sequence $\left(z_{j}\right) \subset S_{Z}=\{z \in Z$ : $\|z\|=1\}$ is called a $\bar{\delta}$-block sequence of $\left(E_{n}\right)$ or a $\bar{\delta}$-skipped block sequence of $\left(E_{n}\right)$ if there are $k_{1}<k_{2}<\ldots$ in $\mathbb{N}$ so that $\left(k_{0}=1\right)$

$$
\left\|z_{n}-P_{\left[k_{n-1}, k_{n}\right)}^{E}\left(z_{n}\right)\right\|<\delta_{n}, \text { or }\left\|z_{n}-P_{\left(k_{n-1}, k_{n}\right)}^{E}\left(z_{n}\right)\right\|<\delta_{n} \text {, respectively, }
$$

for all $n \in \mathbb{N}$ (or $n \leq$ length $\left(z_{j}\right)$ ). Of course one could generalize the notion of $\bar{\delta}$ - block and $\bar{\delta}$-skipped block sequences to more general sequences, but we prefer to introduce this notion only for normalized sequences.

Remark. If $\left(F_{i}\right)$ is a blocking of $\left(E_{i}\right)$ and if $\left(x_{i}\right)$ is a $\bar{\delta}$-skipped block sequence of $\left(F_{i}\right)$, then $\left(x_{i}\right)$ is not necessarily a $\bar{\delta}$-skipped block sequence of $\left(E_{i}\right)$ (since in the definition of skipped block sequence we skip exactly one coordinate). Nevertheless it is clear that $\left(x_{i}\right)$ is a $2 K \bar{\delta}$-skipped block sequence of $\left(E_{i}\right)$, where $K$ is the projection constant of $\left(E_{i}\right)$ in $Z$.

A sequence of finite-dimensional spaces $\left(G_{n}\right)$ is called a blocking of $\left(E_{n}\right)$ if there are $0=k_{0}<k_{1}<k_{2}<\ldots$ in $\mathbb{N}$ so that $G_{n}=\oplus_{i=k_{n-1}+1}^{k_{n}} E_{i}$ for $n=1,2, \ldots$

Definition. For two normalized basic sequences $\left(e_{i}\right)$ and $\left(f_{i}\right)$ we say that $\left(f_{i}\right) C$-dominates $\left(e_{i}\right)$ or that $\left(e_{i}\right)$ is $C$-dominated by $\left(f_{i}\right)$, where $C \geq 1$, if for all $\left(a_{i}\right) \in \mathrm{c}_{00}$

$$
\left\|\sum a_{i} e_{i}\right\| \leq C\left\|\sum a_{i} f_{i}\right\| \text {. }
$$

We say that $\left(f_{i}\right)$ dominates $\left(e_{i}\right)$ or that $\left(e_{i}\right)$ is dominated by $\left(f_{i}\right)$ if, for some $C \geq 1,\left(f_{i}\right)$ $C$-dominates $\left(e_{i}\right)$.

Let $V$ be a Banach space with a 1-unconditional and normalized basis $\left(v_{i}\right)$ and let $1 \leq$ $C<\infty$. We say that an FDD $\left(E_{n}\right)$ of a Banach space $Z$ satisfies $C$ - $V$-lower estimates (in $Z$ ) if all normalized block sequences of $\left(E_{n}\right)$ in $Z C$-dominate $\left(v_{i}\right)$, and $\left(E_{n}\right)$ satisfies $C$-V-upper estimates (in $Z$ ) if $\left(v_{i}\right) C$-dominates all normalized block sequences of $\left(E_{i}\right)$ in $Z$. If $U$ is another space with a normalized and 1 -unconditional basis $\left(u_{i}\right)$, we say that $\left(E_{n}\right)$ satisfies $C$ - $(V, U)$-estimates (in $Z$ ) if it satisfies $C$ - $V$-lower estimates and $C$ - $U$-upper estimates.

We say that $\left(E_{n}\right)$ satisfies $V$-lower estimates (in $Z$ ), $U$-upper estimates (in $Z$ ) or $(V, U)$ estimates (in $Z$ ) if there is a constant $C$ so that $\left(E_{n}\right)$ satisfies $C$ - $V$-lower estimates (in $Z$ ), $C$ - $U$-upper estimates (in $Z$ ) or $C$ - $(V, U)$-estimates (in $Z$ ), respectively.

Remark. It is easy to show that if every normalized block sequence of $\left(E_{i}\right)$ in $Z$ dominates $\left(v_{i}\right)$, then $\left(E_{i}\right)$ satisfies $V$-lower estimates in $Z$. A similar remark holds for $U$-upper estimates. 
We define for $\ell \in \mathbb{N}$

$$
T_{\ell}=\left\{\left(n_{1}, n_{2}, \ldots, n_{\ell}\right): n_{1}<n_{2}<\ldots<n_{\ell} \text { are in } \mathbb{N}\right\}
$$

and

$$
T_{\infty}=\bigcup_{\ell=1}^{\infty} T_{\ell} .
$$

If $\alpha=\left(m_{1}, m_{2}, \ldots, m_{\ell}\right) \in T_{\ell}$, we call $\ell$ the length of $\alpha$ and denote it by $|\alpha|$, and $\beta=$ $\left(n_{1}, n_{2}, \ldots, n_{k}\right) \in T_{\infty}$ is called an extension of $\alpha$, or $\alpha$ is called a restriction of $\beta$, if $k \geq \ell$ and $n_{i}=m_{i}$ for $i=1,2, \ldots, \ell$. We then write $\alpha \leq \beta$ and with this order $\left(T_{\infty}, \leq\right)$ is a tree.

A set $S \subset T_{\infty}$ is called well-founded if it is closed under taking restrictions and if it does not contain any infinite chain with respect to $\leq$. Note that this means that the set $\max (S)$ of maximal elements of $S$ is not empty (provided $S \neq \emptyset$ ) and that

$$
S=\left\{\left(n_{1}, n_{2}, \ldots, n_{k}\right): \exists \ell \geq k \exists n_{k}<n_{k+1}<\ldots<n_{\ell} \quad\left(n_{1}, \ldots, n_{\ell}\right) \in \max (S)\right\} .
$$

In this work trees in a Banach space $X$ are families in $X$ indexed by $T_{\infty}$, and thus they are countable infinitely branching trees of countably infinite length. In Section 4 we will consider families in $X$ indexed by $T_{\ell}$ for some $\ell \in \mathbb{N}$, and we refer to them as trees of length $\ell$.

We introduce the following notation only for trees of infinite length, but note that they can be similarly defined for trees of finite length.

For a tree $\left(x_{\alpha}\right)_{\alpha \in T_{\infty}}$ in a Banach space $X$, and $\alpha=\left(n_{1}, n_{2}, \ldots, n_{\ell}\right) \in T_{\infty} \cup\{\emptyset\}$ we call the sequences of the form $\left(x_{(\alpha, n)}\right)_{n>n_{\ell}}$ nodes of $\left(x_{\alpha}\right)_{\alpha \in T_{\infty}}$. The sequences $\left(y_{n}\right)$ with $y_{i}=x_{\left(n_{1}, n_{2}, \ldots, n_{i}\right)}$ for $i \in \mathbb{N}$ and for some strictly increasing sequence $\left(n_{i}\right) \subset \mathbb{N}$ are called branches of $\left(x_{\alpha}\right)_{\alpha \in T_{\infty}}$. Thus branches of a tree $\left(x_{\alpha}\right)_{\alpha \in T_{\infty}}$ are sequences of the form $\left(x_{\alpha_{n}}\right)$, where $\left(\alpha_{n}\right)$ is an increasing (with respect to extension) sequence in $T_{\infty}$ with $\left|\alpha_{n}\right|=n$ for all $n \in \mathbb{N}$.

If $\left(x_{\alpha}\right)_{\alpha \in T_{\infty}}$ is a tree in $X$ and if $T^{\prime} \subset T_{\infty}$ is closed under taking restrictions so that for each $\alpha \in T^{\prime} \cup\{\emptyset\}$ infinitely many direct successors of $\alpha$ are also in $T^{\prime}$, then we call $\left(x_{\alpha}\right)_{\alpha \in T^{\prime}}$ a full subtree of $\left(x_{\alpha}\right)_{\alpha \in T_{\infty}}$. Note that $\left(x_{\alpha}\right)_{\alpha \in T^{\prime}}$ could then be relabeled to a family indexed by $T_{\infty}$ and note that the branches of $\left(x_{\alpha}\right)_{\alpha \in T^{\prime}}$ are branches of $\left(x_{\alpha}\right)_{\alpha \in T_{\infty}}$ and that the nodes of $\left(x_{\alpha}\right)_{\alpha \in T^{\prime}}$ are subsequences of certain nodes of $\left(x_{\alpha}\right)_{\alpha \in T_{\infty}}$.

We call a tree $\left(x_{\alpha}\right)_{\alpha \in T_{\infty}}$ in a Banach space $X$ normalized if $\left\|x_{\alpha}\right\|=1$ for all $\alpha \in T_{\infty}$ and weakly null if every node is weakly null. If $\left(x_{\alpha}\right)_{\alpha \in T_{\infty}}$ is a tree in a Banach space $Z$ which has an FDD $\left(E_{n}\right)$, then we call it a block tree of $\left(E_{n}\right)$ if every node is a block sequence of $\left(E_{n}\right)$.

We shall need a coordinate-free version of lower and upper estimates.

Definition. Let $V$ be a Banach space with a 1-unconditional and normalized basis $\left(v_{i}\right)$ and let $1 \leq C<\infty$. We say that a Banach space $X$ satisfies $C$ - $V$-lower tree estimates if every normalized weakly null tree $\left(x_{\alpha}\right)_{\alpha \in T_{\infty}}$ in $X$ has a branch $\left(y_{i}\right)$ which $C$-dominates the basis $\left(v_{i}\right)$. Of course we defined domination for basic sequences only but since every normalized, weakly null tree in $X$ admits a full subtree with all branches 2-basic, say, this does not constitute a problem.

We say that $X$ satisfies $C$-V-upper tree estimates if every normalized weakly null tree $\left(x_{\alpha}\right)_{\alpha \in T_{\infty}}$ in $X$ has a branch $\left(y_{i}\right)$ which is $C$-dominated by $\left(v_{i}\right)$.

If $U$ is a second space with a 1-unconditional and normalized basis $\left(u_{i}\right)$, we say that $X$ satisfies $C$ - $(V, U)$-tree estimates if it satisfies $C$ - $V$-lower and $C$ - $U$-upper tree estimates. 
We say that $X$ satisfies $V$-lower tree, $U$-upper tree or $(V, U)$-tree estimates if for some $1 \leq$ $C<\infty X$ satisfies $C$ - $V$-lower tree, $C$ - $U$-upper tree or $C$ - $(V, U)$-tree estimates, respectively.

Proposition 1.1. Let $U$ and $V$ be Banach spaces with normalized, 1-unconditional bases $\left(u_{i}\right)$ and $\left(v_{i}\right)$, respectively. For an infinite subset $N \subset \mathbb{N}$ we let $U^{(N)}$ and $V^{(N)}$ be the closed subspaces spanned by $\left(u_{i}\right)_{i \in N}$ and $\left(v_{i}\right)_{i \in N}$, respectively.

If $C \geq 1$ and a Banach space $X$ satisfies $C$ - $(V, U)$-tree estimates, then it also satisfies $C$ $\left(V^{(N)}, U^{(N)}\right)$-tree estimates (with respect to the 1-unconditional bases $\left(v_{i}\right)_{i \in N}$ and $\left(u_{i}\right)_{i \in N}$ ) for any infinite $N$.

Proof. Let $n_{1}<n_{2}<n_{3}<\ldots$ be such that $N=\left\{n_{1}, n_{2}, \ldots\right\}$, and let $\left(x_{\alpha}\right)_{\alpha \in T_{\infty}}$ be a normalized, weakly null tree in $X$. Let $\left(z_{n}\right)$ be any weakly null sequence in $S_{X}$ (e.g. the top node of $\left.\left(x_{\alpha}\right)_{\alpha \in T_{\infty}}\right)$.

We now consider the following tree $\left(\tilde{x}_{\alpha}\right)_{\alpha \in T_{\infty}}$ which, up to finitely many elements of each node, is an expansion of $\left(x_{\alpha}\right)_{\alpha \in T_{\infty}}$ : for $\alpha=\left(k_{1}, k_{2}, \ldots, k_{\ell}\right) \in T_{\infty}$ we define

$$
\tilde{x}_{\alpha}= \begin{cases}x_{\left(k_{n_{1}}, k_{n_{2}}, \ldots, k_{n_{i}}\right)} & \text { if } \ell=n_{i} \text { for some } i \in \mathbb{N} \\ z_{k_{\ell}} & \text { if } \ell \in \mathbb{N} \backslash N .\end{cases}
$$

Our claim now follows from the fact that $\left(\tilde{x}_{\alpha}\right)_{\alpha \in T_{\infty}}$ is also a normalized, weakly null tree and that for any branch $\left(y_{i}\right)$ of $\left(\tilde{x}_{\alpha}\right)_{\alpha \in T_{\infty}}$ the subsequence $\left(y_{n_{i}}\right)$ is a branch of $\left(x_{\alpha}\right)_{\alpha \in T_{\infty}}$.

In the definition of $U$-upper and $V$-lower tree estimates it is actually not necessary to assume that $C$ exists uniformly for all trees as the following proposition shows.

Proposition 1.2. Let $U$ and $V$ be Banach spaces with normalized, 1-unconditional bases $\left(u_{i}\right)$ and $\left(v_{i}\right)$. Assume that $X$ is a Banach space with the property that every normalized, weakly null tree in $X$ has a branch which dominates $\left(v_{i}\right)$ and a branch which is dominated by $\left(u_{i}\right)$. Then $X$ satisfies $(V, U)$-tree estimates.

Proof. Assume that $X$ has the property that for any $C \geq 1$ there is a weakly null tree in $S_{X}$ so that every branch does not $C$-dominate $\left(v_{i}\right)$ (the argument for $U$ is similar). We will choose a tree $\left(x_{\alpha}\right)_{\alpha \in T_{\infty}}$ which has the property that each branch of $\left(x_{\alpha}\right)_{\alpha \in T_{\infty}}$ does not dominate $\left(v_{i}\right)$.

By induction we will choose for every $m \in \mathbb{N}$ a well-founded subset $S_{m} \subset T_{\infty}$ and a family $\left(x_{\alpha}^{(m)}\right)_{\alpha \in S_{m}}$ in $S_{X}$ so that

a) $S_{m-1} \subset S_{m}$ (with $S_{0}=\emptyset$ ), and $\max \left(S_{m-1}\right) \cap \max \left(S_{m}\right)=\emptyset$;

b) for any $\alpha=\left(n_{1}, \ldots, n_{\ell}\right) \in S_{m} \cup\{\emptyset\}$ either $\alpha$ is maximal in $S_{m}$ or $S_{m}$ contains all the direct successors $(\alpha, n), n>n_{\ell}$ (put $\ell=n_{\ell}=0$ if $\alpha=\emptyset$ ), of $\alpha$;

c) $\left(x_{(\alpha, n)}^{(m)}\right)_{n>n_{\ell}}$ is a weakly null sequence for any $\alpha=\left(n_{1}, \ldots, n_{\ell}\right) \in S_{m} \backslash \max \left(S_{m}\right)$;

d) if $\alpha=\left(n_{1}, \ldots, n_{\ell}\right) \in \max \left(S_{m}\right)$ and $k \in\{1,2, \ldots, \ell\}$ is such that $\left(n_{1}, n_{2}, \ldots, n_{k}\right) \in$ $\max \left(S_{m-1}\right)(k=0$ if $m=1)$, then the segment $\left(y_{i}\right)_{i=k+1}^{\ell}$, where $y_{i}=x_{\left(n_{1}, n_{2}, \ldots, n_{i}\right)}^{(m)}$ for $i=k+1, k+2, \ldots, \ell$, does not $m$-dominate $\left(v_{i}\right)_{i=k+1}^{\ell}$.

Once we have finished the construction of $S_{m}$ and $\left(x_{\alpha}^{(m)}\right)_{\alpha \in S_{m}}$ we deduce from (a) and (b) that $\bigcup_{m \in \mathbb{N}} S_{m}=T_{\infty}$. For $\alpha \in T_{\infty}$ let $x_{\alpha}=x_{\alpha}^{(m)}$ with $m=m(\alpha)=\min \left\{m^{\prime}: \alpha \in S_{m^{\prime}}\right\}$. Let $\left(y_{n}\right)$ be a branch of $\left(x_{\alpha}\right)_{\alpha \in T_{\infty}}$, say $y_{n}=x_{\alpha_{n}}$ for $n \in \mathbb{N}$ and for some increasing (with respect to extension) sequence $\left(\alpha_{n}\right) \subset T_{\infty}$ with $\left|\alpha_{n}\right|=n$ for $n \in \mathbb{N}$. For $m \in \mathbb{N}$ let 
$\ell_{m}=\max \left\{\ell: \alpha_{\ell} \in S_{m}\right\}$ and deduce from $(\mathrm{d})$ that $\left(y_{i}\right)_{i=\ell_{m-1}+1}^{\ell_{m}}=\left(x_{\alpha_{i}}^{(m)}\right)_{i=\ell_{m-1}+1}^{\ell_{m}}$ does not $m$-dominate $\left(v_{i}\right)_{i=\ell_{m-1}+1}^{\ell_{m}}$. Thus $\left(y_{n}\right)$ does not dominate $\left(v_{n}\right)$.

Assume we have chosen $S_{m-1}$ and $\left(x_{\alpha}^{(m-1)}\right)_{\alpha \in S_{m-1}}$ for some $m$. For $\alpha \in \max \left(S_{m-1}\right)$ we can choose a normalized weakly null tree $\left(z_{\beta}^{(\alpha)}\right)_{\beta \in T_{\infty}}$ (if $m=1$, and thus $S_{m-1}=\emptyset$, we choose one tree $\left.\left(z_{\beta}\right)_{\beta \in T_{\infty}}\right)$ so that no branch $3(|\alpha|+m)$-dominates $\left(v_{i}\right)$. Since $\left(z_{\beta}^{(\alpha)}\right)_{\beta \in T_{\infty}}$ is weakly null we can, after passing to an appropriate full subtree, assume that every branch of $\left(z_{\beta}^{(\alpha)}\right)_{\beta \in T_{\infty}}$ is a basic sequence with projection constant less than 3 .

For $\beta=\left(n_{1}, n_{2}, \ldots, n_{\ell}\right) \in T_{\infty} \backslash S_{m-1}$ we define $\alpha(\beta)$ to be the maximal restriction of $\beta$ which lies in $S_{m-1}$ and let $y_{i}^{(\beta)}=z_{\left(n_{1}, n_{2}, \ldots, n_{i}\right)}^{(\alpha(\beta))}$ for $i=1, \ldots, \ell$. Define

$$
S_{m}=S_{m-1} \cup\left\{\beta \in T_{\infty} \backslash S_{m-1}:\left(y_{i}^{(\beta)}\right)_{i=1}^{|\beta|-1} 3(|\alpha(\beta)|+m) \text {-dominates }\left(v_{i}\right)_{i=1}^{|\beta|-1}\right\} .
$$

$S_{m}$ is well-founded, otherwise for some $\alpha \in \max \left(S_{m-1}\right)$ some branch of $\left(z_{\beta}^{(\alpha)}\right)_{\beta \in T_{\infty}}$ would $3(|\alpha|+m)$-dominate $\left(v_{i}\right)$. Since for any $\beta \in T_{\infty} \backslash S_{m-1}$ the sequence $\left(y_{i}^{(\beta)}\right)_{i=1}^{|\alpha(\beta)|} 3|\alpha(\beta)|$ dominates $\left(v_{i}\right)_{i=1}^{|\alpha(\beta)|}$ (a) holds.

If $\beta \in S_{m} \backslash S_{m-1}$ is not maximal in $S_{m}$, then $\left(y_{i}^{(\beta)}\right)_{i=1}^{|\beta|} 3(|\alpha(\beta)|+m)$-dominates $\left(v_{i}\right)_{i=1}^{|\beta|}$. It follows that any direct successor of $\beta$ is in $S_{m}$, which implies condition (b). Finally we put for $\gamma \in S_{m}$

$$
x_{\gamma}^{(m)}= \begin{cases}x_{\gamma}^{(m-1)} & \text { if } \gamma \in S_{m-1} \\ z_{\gamma}^{(\alpha(\gamma))} & \text { if } \gamma \notin S_{m-1} .\end{cases}
$$

Condition (c) is satisfied and, for $\beta \in \max \left(S_{m}\right) \subset T_{\infty} \backslash S_{m-1}$, if $\left(y_{i}^{(\beta)}\right)_{i=|\alpha(\beta)|+1}^{|\beta|} m$-dominates $\left(v_{i}\right)_{i=|\alpha(\beta)|+1}^{|\beta|}$, then $\left(y_{i}^{(\beta)}\right)_{i=1}^{|\beta|} 3(|\alpha(\beta)|+m)$-dominates $\left(v_{i}\right)_{i=1}^{|\beta|}$, which is not true. Thus $(\mathrm{d})$ holds.

The following Proposition generalizes a result of Prus $[\mathrm{P}]$.

Proposition 1.3. Assume that $Z$ has an FDD $\left(E_{i}\right)$, and let $V$ be a space with a normalized and 1-unconditional basis $\left(v_{i}\right)$.

The following statements are equivalent:

a) $\left(E_{i}\right)$ satisfies $V$-lower estimates in $Z$,

b) $\left(E_{i}^{*}\right)$ satisfies $V^{(*)}$-upper estimates in $Z^{(*)}$.

$\left(\right.$ Here $V^{(*)}$-upper estimates are with respect to $\left(v_{i}^{*}\right)$, the sequence of biorthogonal functionals to $\left.\left(v_{i}\right)\right)$.

Moreover, if $\left(E_{i}\right)$ is bimonotone in $Z$, then the equivalence holds true if one replaces, for some $C \geq 1, V$-lower estimates by $C$-V-lower estimates in (a) and $V^{(*)}$-upper estimates by $C-V^{(*)}$-upper estimates in (b).

Remark. By duality Proposition 1.3 holds if we interchange the words lower and upper in (a) and (b).

Proof. W.l.o.g. we assume that $E=\left(E_{i}\right)$ is bimonotone in $Z$.

"(a) $\Rightarrow(\mathrm{b})$ " Assume that $\left(E_{i}\right)$ satisfies $C$ - $V$-lower estimates in $Z$, and let $\left(z_{i}^{*}\right)_{i=1}^{\ell}$ be a block sequence of $E^{*}=\left(E_{i}^{*}\right)$. 
For an appropriate $z \in S_{Z}$ with $\operatorname{supp}_{E}(z) \subset\left[\min \operatorname{supp}_{E^{*}}\left(z_{1}^{*}\right), \max \operatorname{supp}_{E^{*}}\left(z_{l}^{*}\right)\right]$ we have (putting $\max \operatorname{supp}_{E^{*}}\left(z_{0}^{*}\right)=0$ )

$$
\begin{aligned}
\left\|\sum_{i=1}^{\ell} z_{i}^{*}\right\|_{Z^{(*)}} & =\sum_{i=1}^{\ell} z_{i}^{*}(z) \\
& =\sum_{i=1}^{\ell} z_{i}^{*}\left(P_{\left(\max \operatorname{supp}_{E^{*}}\left(z_{i-1}^{*}\right), \max \operatorname{supp}_{E^{*}}\left(z_{i}^{*}\right)\right]}^{E}(z)\right) \\
& \leq \sum_{i=1}^{\ell}\left\|z_{i}^{*}\right\| \cdot\left\|P_{\left(\max \operatorname{supp}_{E^{*}}\left(z_{i-1}^{*}\right), \max \operatorname{supp}_{E^{*}}\left(z_{i}^{*}\right)\right]}^{E}(z)\right\| \\
& \leq\left\|\sum_{i=1}^{\ell}\right\| z_{i}^{*}\left\|\cdot v_{i}^{*}\right\|_{V^{(*)}}\left\|\sum_{i=1}^{\ell}\right\| P_{\left(\max \operatorname{supp}_{E^{*}}\left(z_{i-1}^{*}\right), \max \operatorname{supp}_{E^{*}}\left(z_{i}^{*}\right)\right]}^{E}(z)\left\|v_{i}\right\|_{V} \\
& \leq C\left\|\sum_{i=1}^{\ell}\right\| z_{i}^{*}\left\|\cdot v_{i}^{*}\right\|_{V^{(*)}}\left\|\sum_{i=1}^{\ell}\right\| P_{\left(\max \operatorname{supp}_{E^{*}}\left(z_{i-1}^{*}\right), \max \operatorname{supp}_{E^{*}}\left(z_{i}^{*}\right)\right]}^{E}(z)\left\|z_{i}\right\| \\
& \left(\text { where } z_{i}=\frac{P_{\left(\max \operatorname{supp}_{E^{*}}\left(z_{i-1}^{*}\right), \max \operatorname{supp}_{E^{*}}\left(z_{i}^{*}\right)\right]}^{E}(z)}{\left\|P_{\left(\max \sup _{E^{*}}\left(z_{i-1}^{*}\right), \max \operatorname{supp}_{E^{*}}\left(z_{i}^{*}\right)\right]}(z)\right\|} \text { for } i=1, \ldots, \ell \text { with } \frac{0}{0}=0\right) \\
& =C\left\|\sum_{i=1}^{\ell}\right\| z_{i}^{*}\left\|\cdot v_{i}^{*}\right\|_{V^{(*)}}\|z\|=C\left\|\sum_{i=1}^{\ell}\right\| z_{i}^{*}\left\|\cdot v_{i}^{*}\right\|_{V^{(*)}} \cdot
\end{aligned}
$$

This implies (b).

"(b) $\Rightarrow\left(\right.$ a)" Assume that $\left(E_{i}^{*}\right)$ satisfies $C-V^{(*)}$-upper estimates in $Z^{(*)}$, and let $\left(z_{i}\right)_{i=1}^{\ell}$ be a block sequence of $\left(E_{i}\right)$. Choose $\sum_{i=1}^{\ell} a_{i} v_{i}^{*} \in S_{V^{(*)}}$ so that

$$
\sum_{i=1}^{\ell} a_{i}\left\|z_{i}\right\|=\left(\sum_{i=1}^{\ell} a_{i} v_{i}^{*}\right)\left(\sum_{i=1}^{\ell}\left\|z_{i}\right\| v_{i}\right)=\left\|\sum_{i=1}^{\ell}\right\| z_{i}\left\|v_{i}\right\|_{V},
$$

and choose, for $i=1,2, \ldots, \ell$, a vector $z_{i}^{*} \in S_{Z^{(*)}}$ with $z_{i}^{*}\left(z_{i}\right)=\left\|z_{i}\right\|$ and

$$
\operatorname{supp}_{E^{*}}\left(z_{i}^{*}\right) \subset\left[\min \operatorname{supp}_{E}\left(z_{i}\right), \max \operatorname{supp}_{E}\left(z_{i}\right)\right] .
$$

$\left(\left(E_{i}\right)\right.$ is assumed to be bimonotone in $Z$.) Let $z^{*}=\sum_{i=1}^{\ell} a_{i} z_{i}^{*}$. It follows from our assumption (b) that $\left\|z^{*}\right\| \leq C$, and thus that

$$
\left\|\sum_{i=1}^{\ell} z_{i}\right\| \geq \frac{1}{C} \sum_{i=1}^{\ell} z^{*}\left(z_{i}\right)=\frac{1}{C} \sum_{i=1}^{\ell} a_{i}\left\|z_{i}\right\|=\frac{1}{C}\left\|\sum_{i=1}^{\ell}\right\| z_{i}\left\|v_{i}\right\|_{V},
$$

which implies (a).

Proposition 1.4. Assume that $U$ is a space with a normalized and 1-unconditional basis $\left(u_{i}\right)$ and that $X$ is a reflexive space which satisfies $C$ - $U$-upper tree estimates for some $C \geq 1$.

Then, for any $\varepsilon>0, X^{*}$ satisfies $(2 C+\varepsilon)-U^{(*)}$-lower tree estimates.

Remark. One might ask, whether or not the converse of Proposition 1.4 is true, i.e., similar to the FDD case, whether $X$ satisfies $U$-upper tree estimates if $X^{*}$ satisfies $U^{(*)}$-lower tree estimates. 
The answer is affirmative under certain conditions on $U$, but we do not give a direct proof for that fact. Once we have shown that, under appropriate conditions, a separable, reflexive space $X$ which satisfies $U$-lower tree estimates is both a subspace and a quotient of spaces having an FDD satisfying $U$-lower estimates, this result will follow easily (see Corollary 3.3 in Section 3).

Proof. Let $\eta>0$ and let $\left(x_{i}^{*}\right)$ be a normalized, weakly null sequence in $X^{*}$. Then there is a subsequence $\left(x_{i_{n}}^{*}\right)$ and a normalized, weakly null sequence $\left(y_{n}\right)$ in $X$ so that $x_{i_{n}}^{*}\left(y_{n}\right)>\frac{1}{2+\eta}$ for $n \in \mathbb{N}$. Indeed, for each $i \in \mathbb{N}$ choose $x_{i} \in S_{X}$ with $x_{i}^{*}\left(x_{i}\right)=1$, take a subsequence $\left(x_{j_{n}}\right)$ so that $x=w$ - $\lim _{n \rightarrow \infty} x_{j_{n}}$ exists, and then for an appropriately large $n_{0}$ and for each $n \in \mathbb{N}$ let

and $i_{n}=j_{n+n_{0}}$.

$$
y_{n}=\frac{x_{j_{n+n_{0}}}-x}{\left\|x_{j_{n+n_{0}}}-x\right\|}
$$

Now let $\left(x_{\alpha}^{*}\right)_{\alpha \in T_{\infty}}$ be a normalized, weakly null tree in $X^{*}$. By replacing certain nodes by subsequences, using the previous observation, we can pass to a full subtree $\left(\tilde{x}_{\alpha}^{*}\right)_{\alpha \in T_{\infty}}$ for which there is a normalized, weakly null tree $\left(y_{\alpha}\right)_{\alpha \in T_{\infty}}$ with $\tilde{x}_{\alpha}^{*}\left(y_{\alpha}\right)>\frac{1}{2+\eta}$ for all $\alpha \in T_{\infty}$. Secondly, we may assume, again after passing to full subtrees, that $\left|\tilde{x}_{\alpha}^{*}\left(y_{\beta}\right)\right|<2^{-m-n} \eta$ and $\left|\tilde{x}_{\beta}^{*}\left(y_{\alpha}\right)\right|<2^{-m-n} \eta$ whenever $\alpha, \beta \in T_{\infty},|\alpha|=m<|\beta|=n$ and $\beta$ is an extension of $\alpha$.

By our assumption we can extract a branch $\left(z_{n}\right)$ from $\left(y_{\alpha}\right)_{\alpha \in T_{\infty}}$ which is $C$-dominated by $\left(u_{i}\right)$. Let $\left(z_{n}^{*}\right)$ be the corresponding branch of $\left(\tilde{x}_{\alpha}^{*}\right)_{\alpha \in T_{\infty}}$, and let $\left(a_{i}\right) \in \mathrm{c}_{00}$. Choose $\left(b_{i}\right) \in \mathrm{c}_{00}$ with $\left\|\sum b_{i} u_{i}\right\|=1$ and $\sum a_{i} b_{i}=\left(\sum a_{i} u_{i}^{*}\right)\left(\sum b_{i} u_{i}\right)=\left\|\sum a_{i} u_{i}^{*}\right\|$. It follows that $\left\|\sum b_{i} z_{i}\right\| \leq C$, and thus that (note that $a_{i} b_{i} \geq 0$ for $i \in \mathbb{N}$ )

$$
\begin{aligned}
\left\|\sum a_{i} z_{i}^{*}\right\| & \geq \frac{1}{C} \sum a_{i} z_{i}^{*}\left(\sum b_{i} z_{i}\right) \\
& \geq \frac{1}{C} \sum a_{i} b_{i} z_{i}^{*}\left(z_{i}\right)-\frac{1}{C} \sum_{i \neq j}\left|a_{i} b_{j}\right| \cdot\left|z_{i}^{*}\left(z_{j}\right)\right| \geq \frac{1}{C} \frac{1}{2+\eta} \sum a_{i} b_{i}-\frac{1}{C} \max _{i, j}\left|a_{i} b_{j}\right| \eta,
\end{aligned}
$$

which implies our claim if we choose $\eta>0$ small enough.

The following connection between lower and upper tree estimates and lower and upper estimates for spaces with FDDs will be shown with techniques developed in OS1 and KOS].

Proposition 1.5. Assume that $V$ is a Banach space with a normalized and 1-unconditional basis $\left(v_{i}\right)$, and let $Z$ be a reflexive space with an FDD $\left(E_{i}\right)$.

If $Z$ satisfies $V$-upper or -lower tree estimates, then $\left(E_{i}\right)$ can be blocked into an FDD $\left(F_{i}\right)$ which satisfies $V$-upper or-lower estimates in $Z$.

For the proof of Proposition 1.5 we will need to recall some notation and a proposition from OS1 and OS2.

Definition. If $\mathcal{A} \subseteq S_{X}^{\omega}$, the set of all normalized sequences in $X$, and $\varepsilon>0$, we set

$$
\mathcal{A}_{\varepsilon}=\left\{\left(x_{n}\right) \in S_{X}^{\omega}: \text { there exists }\left(y_{n}\right) \in \mathcal{A} \text { with }\left\|x_{n}-y_{n}\right\|<\frac{\varepsilon}{2^{n}} \text { for all } n\right\} .
$$

$\overline{\mathcal{A}_{\varepsilon}}$ denotes the closure of $\mathcal{A}_{\varepsilon}$ w.r.t. the product topology of the discrete topology on $S_{X}$.

The next result follows from Proposition 2.4 in OS2 (which is a restatement of a part of Theorem 3.3 in OS1) and Proposition 2.5 in OS2. In this section we will only use it for the (much simpler) case $X=Z$. In section 3 we will use it in its full generality. 
Proposition 1.6. Let $X$ be a Banach space which is a subspace of a reflexive space $Z$ with an $F D D\left(E_{i}\right)$. Let $\mathcal{A} \subseteq S_{X}^{\omega}$. Then the following are equivalent.

a) For all $\varepsilon>0$ every normalized, weakly null tree in $X$ has a branch in $\overline{\mathcal{A}_{\varepsilon}}$.

b) For all $\varepsilon>0$ there exists a blocking $\left(F_{i}\right)$ of $\left(E_{i}\right)$ and $\bar{\delta}=\left(\delta_{i}\right), \delta_{i} \downarrow 0$, so that if $\left(x_{n}\right) \subseteq S_{X}$ is a $\bar{\delta}$-skipped block sequence of $\left(F_{i}\right)$ in $Z$, then $\left(x_{n}\right) \in \overline{\mathcal{A}_{\varepsilon}}$.

Proof of Proposition 1.5. If $Z$ satisfies $V$-upper tree estimates, then $Z^{*}$ satisfies, by Proposition 1.4 $V^{(*)}$-lower tree estimates, and if we can block $\left(E_{i}^{*}\right)$ into an FDD $\left(H_{i}^{*}\right)$ which satisfies $V^{(*)}$-lower estimates in $Z^{*}$, then, by Proposition 1.3 and the remark following it, $\left(H_{i}\right)$ satisfies $V$-upper estimates in $Z$.

Therefore we need to prove the Proposition only for the case that $Z$ satisfies $V$-lower tree estimates.

Let $K$ be the projection constant of $\left(E_{i}\right)$ in $Z$, and choose $C \geq 1$ such that the space $Z$ satisfies $C$ - $V$-lower tree estimates. Applying the implication "(a) $\Rightarrow(b)$ " of Proposition 1.6 to $X=Z$,

$$
\mathcal{A}=\left\{\left(z_{i}\right) \in S_{Z}^{\omega}:\left(z_{i}\right) C \text {-dominates }\left(v_{i}\right)\right\}
$$

and to an $\varepsilon>0$ small enough so that

$$
\overline{\mathcal{A}_{\varepsilon}} \subset\left\{\left(z_{i}\right) \in S_{Z}^{\omega}:\left(z_{i}\right) 2 C \text {-dominates }\left(v_{i}\right)\right\},
$$

we obtain a blocking $\left(F_{i}\right)$ of $\left(E_{i}\right)$ and a sequence $\bar{\delta}^{\prime}=\left(\delta_{i}^{\prime}\right), \delta_{i}^{\prime} \downarrow 0$, so that every $\bar{\delta}^{\prime}$-skipped block sequence of $\left(F_{i}\right)$ in $Z 2 C$-dominates $\left(v_{i}\right)$. By the remark following the definition of a $\bar{\delta}$-skipped block sequence we may assume, after replacing $\bar{\delta}^{\prime}$ by $\frac{1}{2 K} \bar{\delta}^{\prime}$ if necessary, that in fact every $\bar{\delta}^{\prime}$-skipped block sequence of any subsequent blocking of $\left(F_{i}\right)$ in $Z 2 C$-dominates $\left(v_{i}\right)$.

By Proposition 1.1$] Z$ also satisfies $C$ - $\left[v_{i+1}\right]_{i=1}^{\infty}$-lower tree estimates. Hence we can repeat the above argument to obtain a further blocking $G=\left(G_{i}\right)$ of $\left(F_{i}\right)$ and a sequence $\bar{\delta}=\left(\delta_{i}\right)$, $\delta_{i} \downarrow 0$ and $\delta_{i} \leq \delta_{i}^{\prime}$ for all $i$, so that every $\bar{\delta}$-skipped block sequence of $\left(G_{i}\right)$ in $Z 2 C$ dominates $\left(v_{i}\right)$ and $\left(v_{i+1}\right)$. W.l.o.g. we can assume that $\sum_{i=1}^{\infty} \delta_{i} \leq \frac{1}{32 C}$. Using a result in [J] (see also [KOS, Lemma 4.2) we can block $\left(G_{i}\right)$ into $H=\left(H_{i}\right)$, say $H_{i}=\oplus_{j=N_{i-1}+1}^{N_{i}} G_{j}$ for $i \in \mathbb{N}$, where $0=N_{0}<N_{1}<N_{2}<\ldots$, so that for any $z \in S_{Z}, z=\sum_{j=1}^{\infty} x_{j}$ with $x_{j} \in G_{j}$ for $j \in \mathbb{N}$, and for every $i \in \mathbb{N}$ there is a $t_{i} \in\left(N_{i-1}, N_{i}\right)$ so that

$$
\left\|P_{t_{i}}^{G}(z)\right\|=\left\|x_{t_{i}}\right\|<\delta_{i}^{2}
$$

Assume now that $\left(z_{n}\right)$ is a normalized block sequence of $\left(H_{i}\right)$ in $Z$. We will show that

$$
\left\|\sum a_{i} z_{i}\right\| \geq \frac{1}{16 C} \quad \text { whenever }\left\|\sum a_{i} v_{i}\right\|=1 \text {. }
$$

For $i \in \mathbb{N}$ let $k_{i} \in \mathbb{N}$ such that $z_{i} \in \oplus_{j=k_{i-1}+1}^{k_{i}} H_{j}\left(k_{0}=0\right)$ and choose some $t_{i} \in$ $\left(N_{k_{i}-1}, N_{k_{i}}\right)$ for which $\left\|P_{t_{i}}^{G}\left(z_{i}\right)\right\|<\delta_{i}^{2}$. For $i \in \mathbb{N}$ write $z_{i}=g_{i}+h_{i}$, where

$$
g_{i}=P_{\left[N_{k_{i-1}}+1, t_{i}\right]}^{G}\left(z_{i}\right) \text { and } h_{i}=P_{\left(t_{i}, N_{k_{i}}\right]}^{G}\left(z_{i}\right) \text {, }
$$

and let

We define for $i \in \mathbb{N}$

$$
B=\left\{i \in \mathbb{N}:\left\|a_{i} h_{i}+a_{i+1} g_{i+1}\right\| \geq \delta_{i}\right\}
$$

$$
w_{i}=\frac{a_{i} h_{i}+a_{i+1} g_{i+1}}{\left\|a_{i} h_{i}+a_{i+1} g_{i+1}\right\|}
$$


$(0 / 0=0)$ and

$$
\alpha_{i}= \begin{cases}\left\|a_{i} h_{i}+a_{i+1} g_{i+1}\right\| & \text { if } i \in B \\ 0 & \text { if } i \notin B .\end{cases}
$$

For $i \in \mathbb{N}$ we put $\tilde{w}_{i}=w_{i}$ if $i \in B$ and we let $\tilde{w}_{i}$ be some normalized element in $G_{N_{k_{i}}}$ if $i \in \mathbb{N} \backslash B$. Note that $\left(\tilde{w}_{i}\right)$ is a $\bar{\delta}$-skipped block sequence of $\left(G_{i}\right)$, and we deduce that

$$
\begin{aligned}
\left\|\sum a_{i} z_{i}\right\|=\left\|\sum_{i} a_{i}+a_{i} h_{i}\right\| \\
\quad=\left\|a_{1} g_{1}+\sum_{i=1}^{\infty}\right\| a_{i} h_{i}+a_{i+1} g_{i+1}\left\|w_{i}\right\| \\
\quad \geq\left\|a_{1} g_{1}+\sum_{i \in B}\right\| a_{i} h_{i}+a_{i+1} g_{i+1}\left\|w_{i}\right\|-\frac{1}{32 C} \\
\quad \geq \frac{1}{2}\left[\left|a_{1}\right|\left\|g_{1}\right\|+\left\|\sum_{i \in B}\right\| a_{i} h_{i}+a_{i+1} g_{i+1}\left\|w_{i}\right\|\right]-\frac{1}{32 C} \\
\quad=\frac{1}{2}\left[\left|a_{1}\right|\left\|g_{1}\right\|+\left\|\sum_{i \in \mathbb{N}} \alpha_{i} \tilde{w}_{i}\right\|\right]-\frac{1}{32 C} \\
\quad \geq \frac{1}{8 C}\left[\left|a_{1}\right|\left\|g_{1}\right\|+\left\|\sum_{i \in \mathbb{N}} \alpha_{i} v_{i}\right\|+\left\|\sum_{i \in \mathbb{N}} \alpha_{i} v_{i+1}\right\|\right]-\frac{1}{32 C} \\
\quad \geq \frac{1}{8 C}\left[\left|a_{1}\right|\left\|g_{1}\right\|+\left\|\sum_{i \in \mathbb{N}}\right\| a_{i} h_{i}+a_{i+1} g_{i+1}\left\|v_{i}\right\|+\left\|\sum_{i \in \mathbb{N}}\right\| a_{i} h_{i}+a_{i+1} g_{i+1}\left\|v_{i+1}\right\|\right]-\frac{1}{16 C} \\
\quad \geq \frac{1}{8 C}\left[\left|a_{1}\right|\left\|g_{1}\right\|+\left\|\sum_{i \in \mathbb{N}}\right\| a_{i} h_{i}\left\|v_{i}\right\|+\left\|\sum_{i \in \mathbb{N}}\right\| a_{i+1} g_{i+1}\left\|v_{i+1}\right\|\right]-\frac{1}{16 C} \\
\quad \geq \frac{1}{8 C}\left\|\sum_{i \in \mathbb{N}}\right\| a_{i} h_{i}+a_{i} g_{i}\left\|v_{i}\right\|-\frac{1}{16 C} \\
\quad=\frac{1}{8 C}\left\|\sum_{i \in \mathbb{N}} a_{i} v_{i}\right\|-\frac{1}{16 C}=\frac{1}{16 C},
\end{aligned}
$$

which finishes the proof of our claim.

\section{THE SPACE $Z_{V}(E)$}

Let $Z$ be a space with an FDD $E=\left(E_{i}\right)$, and let $V$ be a space with a 1 -unconditional and normalized basis $\left(v_{i}\right)$. The space $Z_{V}=Z_{V}(E)$ is defined to be the completion of $c_{00}\left(\oplus E_{i}\right)$ with respect to the following norm $\|\cdot\|_{Z_{V}}$.

$$
\|z\|_{Z_{V}}=\max _{\substack{k \in \mathbb{N} \\ 0=n_{0}<n_{1}<n_{2}<\ldots<n_{k}}}\left\|\sum_{j=1}^{k}\right\| P_{\left(n_{j-1}, n_{j}\right]}^{E}(z)\left\|_{Z} \cdot v_{j}\right\|_{V} \quad \text { for } z \in c_{00}\left(\oplus E_{i}\right) .
$$

Note that $\left(E_{i}\right)$ is a monotone FDD in $Z_{V}$, which implies that the projection constant of $\left(E_{i}\right)$ in $Z_{V}$ is at most 2. $\left(E_{i}\right)$ is bimonotone in $Z_{V}$ if it is bimonotone in $Z$, and if $Z$ and $Z^{\prime}$ are isomorphic and $E^{\prime}=\left(E_{i}^{\prime}\right)$ is the image of $E$ under an isomorphism, then $Z_{V}^{\prime}\left(E^{\prime}\right)$ and $Z_{V}(E)$ are naturally isomorphic. 
Lemma 2.1. Assume that $V$ is a Banach space with a normalized and 1-unconditional basis $\left(v_{i}\right)$ and that $\left(v_{i+1}\right) C$-dominates $\left(v_{i}\right)$ for some $C \geq 1$. Let $Z$ be a space with an FDD $E=\left(E_{i}\right)$.

Then every normalized block sequence $\left(z_{n}\right)$ of $\left(E_{i}\right)$ in $Z_{V}(E)$ has a subsequence $\left(z_{i_{n}}\right)$ for which there is a normalized block sequence $\left(b_{n}\right)$ in $V$ so that for some $d>0$

$$
\left\|\sum_{i=1}^{\infty} a_{i} z_{i}\right\|_{Z_{V}} \geq d\left\|\sum_{n=1}^{\infty} a_{i_{n}} b_{n}\right\|_{V} \text { whenever }\left(a_{i}\right) \in \mathrm{c}_{00} .
$$

In particular $\left(z_{i_{n}}\right)$ dominates $\left(b_{n}\right)$ (choose $a_{i}=0$ if $i \notin\left\{i_{1}, i_{2}, \ldots\right\}$ ).

Proof. We assume without loss of generality that $\left(E_{i}\right)$ is bimonotone in $Z$. Let $\left(z_{i}\right)$ be a normalized block sequence of $\left(E_{i}\right)$ in $Z_{V}$. We will choose a subsequence $\left(z_{i_{j}}\right)_{j=1}^{\infty}$ together with $\varepsilon_{0}>0$ and increasing sequences $\left(m_{j}\right)_{j=1}^{\infty}$ and $\left(n_{s}\right)_{s=1}^{\infty}$ in $\mathbb{N}$ so that for all $j \in \mathbb{N}$

$$
n_{m_{j}}=\max \operatorname{supp}_{E}\left(z_{i_{j}}\right) \text {, and }
$$

$$
\left\|\sum_{s=m_{j-1}+1}^{m_{j}}\right\| P_{\left(n_{s-1}, n_{s}\right]}^{E}\left(z_{i_{j}}\right)\left\|_{Z} \cdot v_{s}\right\|_{V} \geq \varepsilon_{0} \quad\left(\text { where } m_{0}=n_{0}=0\right) .
$$

Then (3) follows immediately with $b_{j}=\tilde{b}_{j} /\left\|\tilde{b}_{j}\right\|$ and

$$
\tilde{b}_{j}=\sum_{s=m_{j-1}+1}^{m_{j}}\left\|P_{\left(n_{s-1}, n_{s}\right]}^{E}\left(z_{i_{j}}\right)\right\|_{Z} \cdot v_{s} \quad \text { for } j \in \mathbb{N} .
$$

Indeed, if $\left(a_{i}\right) \in c_{00}$ and $z=\sum a_{i} z_{i}$, then

$$
\begin{aligned}
\|z\|_{Z_{V}} & \geq\left\|\sum_{j=1}^{\infty} \sum_{s=m_{j-1}+1}^{m_{j}}\right\| P_{\left(n_{s-1}, n_{s}\right]}^{E}(z)\left\|_{Z} \cdot v_{s}\right\|_{V} \\
& \geq\left\|\sum_{j=1}^{\infty} \sum_{s=m_{j-1}+1}^{m_{j}}\left|a_{i_{j}}\right| \cdot\right\| P_{\left(n_{s-1}, n_{s}\right]}^{E}\left(z_{i_{j}}\right)\left\|_{Z} \cdot v_{s}\right\|_{V}
\end{aligned}
$$

(using bimonotonicity)

$$
=\left\|\sum a_{i_{j}} \tilde{b}_{j}\right\|_{V} \geq \varepsilon_{0}\left\|\sum a_{i_{j}} b_{j}\right\|_{V} .
$$

We can, for each $i \in \mathbb{N}$, choose positive integers $k(i)$ and $0=n_{0}(i)<n_{1}(i)<\ldots<$ $n_{k(i)}(i)=\max \operatorname{supp}_{E}\left(z_{i}\right)$ so that

$$
\left\|z_{i}\right\|_{Z_{V}}=\left\|\sum_{j=1}^{k(i)}\right\| P_{\left(n_{j-1}(i), n_{j}(i)\right]}^{E}\left(z_{i}\right)\left\|_{Z} \cdot v_{j}\right\|_{V}=1 .
$$

We can assume that we are in one of the following three cases:

Case 1: $\left\|z_{i_{n}}\right\|_{Z} \geq \varepsilon_{0}$ for all $n \in \mathbb{N}$, some $\varepsilon_{0}>0$ and some subsequence $\left(z_{i_{n}}\right)$ of $\left(z_{i}\right)$.

Case 2: $\left\|\sum_{j=1}^{\max \operatorname{supp}_{E}\left(z_{i_{n}}\right)}\right\| P_{j}^{E}\left(z_{i_{n}}\right)\left\|_{Z} \cdot v_{j}\right\|_{V} \geq \varepsilon_{0}$ for all $n \in \mathbb{N}$, some $\varepsilon_{0}>0$ and some subsequence $\left(z_{i_{n}}\right)$ of $\left(z_{i}\right)$.

Case 3: $\lim _{i \rightarrow \infty}\left\|z_{i}\right\|_{Z}=0$ and

$$
\lim _{i \rightarrow \infty}\left\|\sum_{j=1}^{\max _{\operatorname{supp}}\left(z_{i}\right)}\right\| P_{j}^{E}\left(z_{i}\right)\left\|_{Z} \cdot v_{j}\right\|_{V}=0 .
$$


Indeed, if all subsequences $\left(z_{i_{n}}\right)$ fail Cases 1 and 2, then Case 3 holds.

In Case 1 we choose $n_{j}=\max \operatorname{supp}\left(z_{i_{j}}\right), m_{j}=j$, and in case 2 we choose $n_{j}=j, m_{j}=$ $\max \operatorname{supp}\left(z_{i_{j}}\right)$ for each $j \in \mathbb{N}$. In case 3 we will choose by induction $i_{j}, m_{j}$ and $n_{m_{j-1}+1}<$ $n_{m_{j-1}+2}<\ldots<n_{m_{j}}, j \in \mathbb{N}$, so that $\left(i_{j}\right),\left(m_{j}\right)$ and $\left(n_{s}\right)$ are increasing and so that (44) and (51) are satisfied with $\varepsilon_{0}=1 / 2$ for all $j \in \mathbb{N}$.

For $j=1$ we choose $i_{1}=1, m_{1}=k(1)$ and $n_{s}=n_{s}(1)$ for $s=1,2, \ldots, m_{1}$. Assume we have chosen $i_{1}<i_{2}<\ldots<i_{j-1}, m_{1}<m_{2}<\ldots<m_{j-1}$ and $n_{1}<\ldots<n_{m_{j-1}}$. By the first condition of Case 3 we can choose $i^{\prime}>i_{j-1}$ large enough so that for all $i \geq i^{\prime}$ there is a $k^{\prime}(i) \in\left(m_{j-1}, k(i)\right)$ with (we are using that $\max _{s \leq k(i)}\left\|P_{\left(n_{s-1}(i), n_{s}(i)\right]}^{E}\left(z_{i}\right)\right\|_{Z} \rightarrow 0$ as $i \rightarrow \infty$ )

$$
1 / 3<\left\|\sum_{s=1}^{k^{\prime}(i)}\right\| P_{\left(n_{s-1}(i), n_{s}(i)\right]}^{E}\left(z_{i}\right)\left\|_{Z} \cdot v_{s}\right\|_{V}<1 / 2
$$

and thus, by (6),

$$
\left\|\sum_{s=k^{\prime}(i)+1}^{k(i)}\right\| P_{\left(n_{s-1}(i), n_{s}(i)\right]}^{E}\left(z_{i}\right)\left\|_{Z} \cdot v_{s}\right\|_{V}>1 / 2 .
$$

Since by our assumption $\left(v_{s_{0}+s}\right)_{s=1}^{\infty} C^{s_{0}}$-dominates $\left(v_{s}\right)_{s=1}^{\infty}$ for all $s_{0} \in \mathbb{N}$, it follows, using the second condition in Case 3, that $n_{k^{\prime}(i)}(i)-k^{\prime}(i) \rightarrow \infty$ as $i \rightarrow \infty$. Indeed, assuming this is not true, we can choose an infinite subset $N \subset \mathbb{N}$ so that for some $M \geq 0$ we have $n_{k^{\prime}(i)}(i)-k^{\prime}(i)=M$ for all $i \in N$. It follows that for each $i \in N$ at most $M$ of the intervals $\left(n_{s-1}(i), n_{s}(i)\right]$ in (7) has length exceeding 1 and that the sum of the lengths of these intervals is at most $2 M$, and hence their contribution to the norm in (7) converges to 0 as $i \rightarrow \infty$. Thus by a further stabilization, replacing $N$ by an infinite subset of $N$ if necessary, we may assume that for some $s_{0} \leq M$ and for all $i \in N$ there is an interval $J_{i} \subset\left\{1,2, \ldots, k^{\prime}(i)\right\}$ such that

$$
\left\|\sum_{j \in J_{i}}\right\| P_{j+s_{0}}^{E}\left(z_{i}\right)\left\|_{Z} \cdot v_{j}\right\|_{V} \geq \frac{1}{3(M+1)+1},
$$

and thus, by our assumption on $\left(v_{j}\right)$, for some $\delta_{0}>0$

$$
\left\|\sum_{j=1}^{\max \operatorname{supp}\left(z_{i}\right)}\right\| P_{j}^{E}\left(z_{i}\right)\left\|_{Z} \cdot v_{j}\right\| \geq\left\|\sum_{j \in J_{i}}\right\| P_{j+s_{0}}^{E}\left(z_{i}\right)\left\|_{Z} \cdot v_{j+s_{0}}\right\| \geq \delta_{0},
$$

which contradicts the second assumption of Case 3 .

Therefore we can choose $i_{j}=i>i^{\prime}$ large enough so that $n_{k^{\prime}(i)}(i)-k^{\prime}(i)>n_{m_{j-1}}$. Then set $m_{j}=k(i)$ and $n_{s}=n_{s}(i)$ for $k^{\prime}(i) \leq s \leq k(i)$, choose $n_{m_{j-1}+1}<n_{m_{j-1}+2}<\ldots<n_{k^{\prime}(i)-1}$ arbitrarily from the set $\left(n_{m_{j-1}}, n_{k^{\prime}(i)}(i)\right)$, and deduce our claim from (8).

Corollary 2.2. Assume that $\left(v_{i}\right)$ is a normalized, boundedly complete and 1-unconditional basis of a Banach space $V$ so that $\left(v_{i+1}\right)$ dominates $\left(v_{i}\right)$, and let $Z$ be a space with an FDD $E=\left(E_{i}\right)$.

Then $\left(E_{i}\right)$ is a boundedly complete FDD for $Z_{V}(E)$.

Proof. Let $\left(z_{n}\right)$ be a normalized block sequence of $\left(E_{i}\right)$ in $Z_{V}$. Choose a subsequence $\left(z_{i_{n}}\right)$ of $\left(z_{n}\right)$ and a normalized block sequence $\left(b_{n}\right)$ in $V$ so that (3) of Lemma 2.1] is satisfied for 
some $d>0$. Then it follows from the assumption that $\left(v_{i}\right)$ is boundedly complete that if $\left(a_{i}\right) \subset[\varepsilon, \infty)$ for some $\varepsilon>0$, then

$$
\left\|\sum_{j=1}^{i_{n}} a_{j} z_{j}\right\|_{Z_{V}} \geq d\left\|\sum_{j=1}^{n} a_{i_{j}} b_{j}\right\|_{V} \rightarrow \infty \quad \text { as } n \rightarrow \infty .
$$

Since $\left(z_{i}\right)$ was an arbitrary normalized block sequence of $\left(E_{i}\right)$ in $Z_{V}$ it follows that $\left(E_{i}\right)$ is boundedly complete in $Z_{V}$.

Lemma 2.3. Let $V$ be a Banach space with a normalized and 1-unconditional basis $\left(v_{i}\right)$ and assume that the space $Z$ has an FDD $E=\left(E_{i}\right)$.

If $\left(v_{i}\right)$ is shrinking and if $\left(E_{i}\right)$ is shrinking in $Z$ then $\left(E_{i}\right)$ is shrinking in $Z_{V}(E)$.

Proof. W.l.o.g. we assume that $\left(E_{i}\right)$ is bimonotone in $Z$ and therefore also in $Z_{V}(E)$. We first note that if $v^{*}=\sum_{i=1}^{\infty} a_{i} v_{i}^{*}$ converges in $V^{*}$ and $\left\|v^{*}\right\| \leq 1$ and if $\left(z_{i}^{*}\right)$ is a normalized block sequence of $\left(E_{i}^{*}\right)$ in $Z^{*}$, then the series $\sum_{i=1}^{\infty} a_{i} z_{i}^{*}$ converges in $\left(Z_{V}\right)^{*}$ and $\left\|\sum_{i=1}^{\infty} a_{i} z_{i}^{*}\right\|_{\left(Z_{V}\right)^{*}} \leq 1$. Indeed, for $m \leq n$ in $\mathbb{N}$ there is a $z \in S_{Z_{V}}$ with $\operatorname{supp}_{E}(z) \subset$ $\left[\min \operatorname{supp}_{E^{*}}\left(z_{m}^{*}\right), \max \operatorname{supp}_{E^{*}}\left(z_{n}^{*}\right)\right]$ so that

$$
\begin{aligned}
\left\|\sum_{i=m}^{n} a_{i} z_{i}^{*}\right\|_{\left(Z_{V}\right)^{*}} & =\sum_{i=m}^{n} a_{i} z_{i}^{*}(z) \\
& \leq \sum_{i=m}^{n}\left|a_{i}\right| \cdot\left\|P_{\left(\max \operatorname{supp}_{E^{*}}\left(z_{i-1}^{*}\right), \max \operatorname{supp}_{E^{*}}\left(z_{i}^{*}\right)\right]}^{E}(z)\right\|_{Z} \\
& \leq\left\|\sum_{i=m}^{n} a_{i} v_{i}^{*}\right\|_{V^{*}} \cdot\left\|\sum_{i=m}^{n}\right\| P_{\left(\max \operatorname{supp}_{E^{*}}\left(z_{i-1}^{*}\right), \max _{\operatorname{supp}} \sup _{E^{*}}\left(z_{i}^{*}\right)\right]}(z)\left\|_{Z} \cdot v_{i}\right\|_{V} \\
& \leq\left\|\sum_{i=m}^{n} a_{i} v_{i}^{*}\right\|_{V^{*}} \cdot\|z\|_{Z_{V}}=\left\|\sum_{i=m}^{n} a_{i} v_{i}^{*}\right\|_{V^{*}},
\end{aligned}
$$

which implies the claim.

Define

$$
\begin{aligned}
K=\{ & \left.\sum_{i=1}^{\infty} a_{i} z_{i}^{*}:\left\|\sum_{i=1}^{\infty} a_{i} v_{i}^{*}\right\|_{V^{*}} \leq 1,\left(z_{i}^{*}\right) \text { is an infinite block sequence in } S_{Z^{*}}\right\} \\
& \cup\left\{\sum_{i=1}^{\ell} a_{i} z_{i}^{*}: \ell \in \mathbb{N},\left\|\sum_{i=1}^{\ell} a_{i} v_{i}^{*}\right\|_{V^{*}} \leq 1,\left(z_{i}^{*}\right) \text { is a block sequence in } S_{Z^{*}} \text { of length } \ell\right\},
\end{aligned}
$$

where we allow in the second of the two sets which form $K$ the last element $z_{\ell}^{*}$ to have infinite support. Clearly, $K$ is a $Z_{V}$-norming subset (isometrically) of $B_{\left(Z_{V}\right)^{*}}$. We claim that $K$ is $w^{*}$-compact. Indeed, let $y_{n}^{*}=\sum_{i=1}^{\infty} a_{i}^{(n)} z_{(n, i)}^{*} \in K$ for $n \in \mathbb{N}$ (where, for $n \in \mathbb{N}$, $a_{i}^{(n)}$ and $z_{(n, i)}^{*}$ may eventually vanish in case that $y_{n}^{*}$ is in the second of the two sets which form $K)$. After passing to a subsequence we can assume that

$$
\begin{aligned}
& z_{i}^{*}=w^{*}-\lim _{n \rightarrow \infty} z_{(n, i)}^{*} \in B_{Z^{*}} \text { exists for all } i \in \mathbb{N} \text { and } \\
& v^{*}=w^{*}-\lim _{n \rightarrow \infty} \sum a_{i}^{(n)} v_{i}^{*} \in B_{V^{*}} \text { exists. }
\end{aligned}
$$

Since $\left(v_{i}\right)$ is a shrinking basis of $V$, we can write $v^{*}=\sum a_{i} v_{i}^{*}$ for some scalars $\left(a_{i}\right)$. 
Note that if $P_{j}^{E^{*}}\left(z_{i}^{*}\right) \neq 0$, then $P_{j^{\prime}}^{E^{*}}\left(z_{i^{\prime}}^{*}\right)=0$ whenever $j^{\prime} \leq j$ and $i^{\prime}>i$ or $j^{\prime} \geq j$ and $i^{\prime}<i$. This means that the non-zero terms of the sequence $\left(z_{i}^{*}\right)$ form a (finite or infinite) block sequence of $\left(E_{i}^{*}\right)$ (where, in the finite case, the last term may have infinite support).

Since $\left\|z_{i}^{*}\right\|_{Z^{*}} \leq 1$ for $i \in \mathbb{N}$, and since $\left(v_{i}\right)$ is 1-unconditional, it follows that

$$
z^{*}=\sum_{i, z_{i}^{*} \neq 0} a_{i}\left\|z_{i}^{*}\right\|_{Z^{*}} \frac{z_{i}^{*}}{\left\|z_{i}^{*}\right\|_{Z^{*}}} \in K .
$$

Finally, for $j \in \mathbb{N}$ and $z \in E_{j}$ we have

$$
\lim _{n \rightarrow \infty} y_{n}^{*}(z)=\sum_{i=1}^{\infty} a_{i} z_{i}^{*}(z)=z^{*}(z),
$$

and thus $z^{*}$ is the $w^{*}$-limit of $\left(y_{n}^{*}\right)$. This shows that $K$ is $w^{*}$-closed.

We deduce that $Z_{V}$ is embedded in $C(K)$, the space of continuous functions on $K$. Let $\left(z_{i}\right)$ be a bounded block sequence of $\left(E_{i}\right)$ in $Z_{V}$, and let $z^{*}=\sum_{i=1}^{\infty} a_{i} z_{i}^{*} \in K$ (i.e. $\left\|\sum_{i=1}^{\infty} a_{i} v_{i}^{*}\right\|_{V^{*}} \leq 1$ and either $\left\|z_{i}^{*}\right\|_{Z^{*}}=1$ for all $i \in \mathbb{N}$ or, for some $\ell \in \mathbb{N},\left\|z_{i}^{*}\right\|_{Z^{*}}=1$ for all $i \leq \ell$ and $z_{i}^{*}=0$ for $i>\ell$ ). If $\left(z_{j}^{*}\right)$ is an infinite normalized block sequence, then it follows that

$$
\begin{aligned}
& z^{*}\left(z_{i}\right)=\sum_{\substack{j \in \mathbb{N}, \max \operatorname{supp}_{E^{*}}\left(z_{j}^{*}\right) \geq \min \operatorname{supp}_{E}\left(z_{i}\right)}} a_{j} z_{j}^{*}\left(z_{i}\right) \\
& \leq\left\|\sum_{\substack{j \in \mathbb{N}, \max \operatorname{supp}_{E^{*}}\left(z_{j}^{*}\right) \geq \min \operatorname{supp}_{E}\left(z_{i}\right)}} a_{j} v_{j}^{*}\right\|_{V^{*}} \rightarrow 0 \quad \text { as } i \rightarrow \infty .
\end{aligned}
$$

If, for some $\ell \in \mathbb{N},\left\|z_{\ell}^{*}\right\|=1$ and $z_{j}^{*}=0$ for $j>\ell$, then from the assumption that $\left(E_{i}\right)$ is shrinking in $Z$ and that $\left(z_{i}\right)$ is a bounded block sequence of $\left(E_{i}\right)$ in $Z_{V}$, and thus also in $Z$, we deduce that for large enough $i \in \mathbb{N}$

$$
z^{*}\left(z_{i}\right)=a_{\ell} z_{\ell}^{*}\left(z_{i}\right) \rightarrow 0 \quad \text { as } i \rightarrow \infty .
$$

It follows that $\left(z_{i}\right)$ is weakly null in $C(K)$, and thus in $Z_{V}$. Since $\left(z_{i}\right)$ was an arbitrary bounded block sequence in $Z_{V}$, this finishes the proof that $\left(E_{i}\right)$ is shrinking in $Z_{V}$.

Corollary 2.2 and Lemma 2.3 yield the following result.

Corollary 2.4. Assume that $Z$ is a space with a shrinking FDD $E=\left(E_{i}\right)$ and that $V$ is a reflexive Banach space with a normalized and 1-unconditional basis $\left(v_{i}\right)$ such that $\left(v_{i+1}\right)$ dominates $\left(v_{i}\right)$. Then $Z_{V}(E)$ is reflexive.

We will now formulate conditions on $V$ which ensure that, given a space $Z$ with an FDD $\left(E_{i}\right)$, every normalized block tree in $Z_{V}(E)$ admits a branch that dominates some normalized block sequence of $\left(v_{i}\right)$. We consider the following two forms of shift invariance of $V$.

Definition. We say that $V$ has the strong right shift property if (SRS) there exists $c>0$ so that for all $\left(a_{i}\right) \in c_{00}$ and all $n \in \mathbb{N}$

$$
\left\|\sum_{i=1}^{\infty} a_{i} v_{i+n}\right\|_{V} \geq c\left\|\sum_{i=1}^{\infty} a_{i} v_{i}\right\|_{V} .
$$

We say that $V$ has the weak left shift property if 
(WLS) there exists $d>0$ so that for all $m \in \mathbb{N}$ there exists $L=L(m) \geq m$ so that for all $k \leq m$

$$
\left\|\sum_{i=L+1}^{\infty} a_{i} v_{i-k}\right\|_{V} \geq d\left\|\sum_{i=L+1}^{\infty} a_{i} v_{i}\right\|_{V} \text { whenever }\left(a_{i}\right) \in \mathrm{c}_{00} .
$$

Lemma 2.5. Let $Z$ be a space with an FDD $E=\left(E_{i}\right)$ and let $V$ be a space with a 1unconditional and normalized basis $\left(v_{i}\right)$.

If $V$ satisfies $(S R S)$ and $(W L S)$, then there is a $C \geq 1$ so that every normalized block tree of $\left(E_{i}\right)$ in $Z_{V}(E)$ has a branch that $C$-dominates some normalized block sequence of $\left(v_{i}\right)$.

Proof. Without loss of generality we can, after renorming $Z$ if necessary, assume that $\left(E_{i}\right)$ is bimonotone. Let $c$ and $d$ be as in (SRS) and (WLS).

Given a block tree in $S_{Z_{V}(E)}$, we can extract a branch $\left(z_{i}\right)$ so that

$$
\begin{aligned}
& L\left(b_{i-1}\right)<a_{i} \text { for all } i>1 \text {, where } a_{i}=\min \operatorname{supp}\left(z_{i}\right) \text { and } \\
& \qquad b_{i}=\max \operatorname{supp}\left(z_{i}\right) \text { for all } i \in \mathbb{N} .
\end{aligned}
$$

Using (SRS) and the fact that $\left(z_{i}\right)$ is normalized in $Z_{V}$, we can choose, for each $i \in \mathbb{N}$, $k(i) \in \mathbb{N}$ and $0=n_{0}(i)<n_{1}(i)<n_{2}(i)<\ldots<n_{k(i)}(i)=b_{i}$ so that $\left(a_{i} \leq k(i)\right.$ and $)$

$$
\begin{aligned}
& n_{j}(i)=j \quad \text { for } j=0,1,2, \ldots, a_{i}-1, \\
& 1=\left\|z_{i}\right\|_{Z_{V}} \geq\left\|\sum_{j=1}^{k(i)}\right\| P_{\left(n_{j-1}(i), n_{j}(i)\right]}^{E}\left(z_{i}\right)\left\|_{Z} \cdot v_{j}\right\|_{V} \geq c .
\end{aligned}
$$

(Note that by forcing (10) we can only achieve the value $c$.)

Put $m_{1}=k(1)$ and $n_{j}=n_{j}(1)$ for $j=0,1,2, \ldots, m_{1}$, and assume that $m_{1}<m_{2}<$ $\ldots<m_{i-1}$ and $n_{0}<n_{1}<\ldots<n_{m_{i-1}}=b_{i-1}$ have been chosen for some $i>1$. We put $m_{i}=m_{i-1}+k(i)-b_{i-1}$ and $n_{j}=n_{j-m_{i-1}+b_{i-1}}(i)$ for $j=m_{i-1}+1, m_{i-1}+2, \ldots, m_{i}$. Note that

$$
\begin{gathered}
m_{i} \geq m_{i-1}+a_{i}-b_{i-1}>m_{i-1}, \\
n_{m_{i-1}}=b_{i-1}=n_{b_{i-1}}(i)<n_{b_{i-1}+1}(i)=n_{m_{i-1}+1} \quad \text { and } \\
n_{m_{i}}=n_{k(i)}(i)=b_{i} .
\end{gathered}
$$

We deduce for $i \in \mathbb{N}$ that

$$
\begin{aligned}
& \left\|\sum_{j=m_{i-1}+1}^{m_{i}}\right\| P_{\left(n_{j-1}, n_{j}\right]}^{E}\left(z_{i}\right)\left\|_{Z} \cdot v_{j}\right\|_{V} \\
& \quad=\left\|\sum_{j=b_{i-1}+1}^{k(i)}\right\| P_{\left(n_{j-1}(i), n_{j}(i)\right]}^{E}\left(z_{i}\right)\left\|_{Z} \cdot v_{j-\left(b_{i-1}-m_{i-1}\right)}\right\|_{V} \\
& =\left\|\sum_{j=a_{i}}^{k(i)}\right\| P_{\left(n_{j-1}(i), n_{j}(i)\right]}^{E}\left(z_{i}\right)\left\|_{Z} \cdot v_{j-\left(b_{i-1}-m_{i-1}\right)}\right\|_{V}
\end{aligned}
$$

(since $P_{\left(n_{j-1}(i), n_{j}(i)\right]}^{E}\left(z_{i}\right)=0$ for $\left.j<a_{i}\right)$ 


$$
\begin{aligned}
\geq d \| & \left\|\sum_{j=a_{i}}^{k(i)}\right\| P_{\left(n_{j-1}(i), n_{j}(i)\right]}^{E}\left(z_{i}\right)\left\|_{Z} \cdot v_{j}\right\|_{V} \geq c d \\
& \quad\left(\text { since } b_{i-1}-m_{i-1} \leq b_{i-1} \text { and } L\left(b_{i-1}\right)<a_{i}\right) .
\end{aligned}
$$

Our claim now follows as in the proof of Lemma 2.1

Lemma 2.6. Let $V$ and $U$ be Banach spaces with normalized and 1-unconditional bases $\left(v_{i}\right)$ and $\left(u_{i}\right)$, respectively, and assume that every subsequence of $\left(u_{i}\right)$ dominates every normalized block sequence of $\left(v_{i}\right)$ and that every subsequence of $\left(v_{i}\right)$ is dominated by every normalized block sequence of $\left(u_{i}\right)$. Let $Z$ be a Banach space with an FDD $\left(E_{i}\right)$.

If $\left(E_{i}\right)$ satisfies $U$-upper estimates in $Z$, then $\left(E_{i}\right)$ also satisfies $U$-upper estimates in $Z_{V}$.

Proof. It follows from the assumptions that for some constants $C_{1}, C_{2}$ and $C_{3}$ in $[1, \infty)$ we have

$$
\left\|\sum_{i=1}^{\infty} z_{i}\right\|_{Z} \leq C_{1}\left\|\sum_{i=1}^{\infty}\right\| z_{i}\left\|_{Z} \cdot u_{i}\right\|_{U} \text { for all block sequences }\left(z_{i}\right) \text { of }\left(E_{i}\right)
$$

subsequences of $\left(v_{i}\right)$ are $C_{2}$-dominated by normalized block sequences of $\left(u_{i}\right)$, normalized block sequences of $\left(v_{i}\right)$ are $C_{3}$-dominated by subsequences of $\left(u_{i}\right)$.

Let $K$ be the projection constant of $\left(E_{i}\right)$ in $Z$ and set $C=C_{3}+C_{1} C_{2}+2 K C_{2}$. We show that for any finite block sequence $\left(z_{i}\right)_{i=1}^{m}$ of $\left(E_{i}\right)$ and for any $k$ and $n_{1}<\ldots<n_{k}$ in $\mathbb{N}$ we have (putting $z=\sum_{i=1}^{m} z_{i}$ and $n_{0}=0$ )

$$
\left\|\sum_{j=1}^{k}\right\| P_{\left(n_{j-1}, n_{j}\right]}^{E}(z)\left\|_{Z} \cdot v_{j}\right\|_{V} \leq C\left\|\sum_{i=1}^{m}\right\| z_{i}\left\|_{Z_{V}} \cdot u_{i}\right\|_{U} .
$$

Taking then the supremum of the left side of (15) over all choices of $k$ and $n_{1}<\ldots<n_{k}$ in $\mathbb{N}$, we obtain

$$
\left\|\sum_{i=1}^{m} z_{i}\right\|_{Z_{V}} \leq C\left\|\sum_{i=1}^{m}\right\| z_{i}\left\|_{Z_{V}} \cdot u_{i}\right\|_{U}
$$

and thus that $\left(E_{i}\right)$ satisfies $C$ - $U$-upper estimates in $Z_{V}$. Note that in proving (15) we can of course assume that $n_{k} \leq \max \operatorname{supp}\left(z_{m}\right)$.

For $i=1,2, \ldots, m$ put

$$
J_{i}=\left\{j \in\{1,2 \ldots, k\}: \max \operatorname{supp}_{E}\left(z_{i-1}\right) \leq n_{j-1}<n_{j} \leq \max _{\operatorname{supp}}\left(z_{i}\right)\right\}
$$

(with $\max \operatorname{supp}_{E}\left(z_{0}\right)=0$ ) and $J_{0}=\{1,2, \ldots, k\} \backslash \bigcup_{i=1}^{m} J_{i}$.

For $j=1,2, \ldots, k$ put

$$
I_{j}=\left\{i \in\{1,2, \ldots, m\}: n_{j-1}<\min \operatorname{supp}_{E}\left(z_{i}\right)<\max \operatorname{supp}_{E}\left(z_{i}\right) \leq n_{j}\right\}
$$

and $I_{0}=\{1,2, \ldots, m\} \backslash \bigcup_{j=1}^{k} I_{j}$.

Firstly, we have

$$
\begin{aligned}
& \left\|\sum_{i=1}^{m} \sum_{j \in J_{i}}\right\| P_{\left(n_{j-1}, n_{j}\right]}^{E}\left(z_{i}\right)\left\|_{Z} \cdot v_{j}\right\|_{V} \\
& \quad=\left\|\sum_{i=1}^{m} b_{i}\right\|_{V} \quad\left(\text { where } b_{i}=\sum_{j \in J_{i}}\left\|P_{\left(n_{j-1}, n_{j}\right]}^{E}\left(z_{i}\right)\right\|_{Z} \cdot v_{j} \quad \text { for } 1 \leq i \leq m\right)
\end{aligned}
$$




$$
\begin{aligned}
& \leq C_{3}\left\|\sum_{i=1}^{m}\right\| b_{i}\left\|_{V} \cdot u_{i}\right\|_{U} \\
& \leq C_{3}\left\|\sum_{i=1}^{m}\right\| z_{i}\left\|_{Z_{V}} \cdot u_{i}\right\|_{U} .
\end{aligned}
$$

(Note that some (or all) of the $b_{i}$ may be zero, however the third line above is still valid using assumption (14).) Secondly,

$$
\begin{aligned}
\left\|\sum_{j \in J_{0}}\right\| \sum_{i \in I_{j}} z_{i}\left\|_{Z} \cdot v_{j}\right\|_{V} & \\
\leq & C_{1}\left\|\sum_{j \in J_{0}}\right\| b_{j}\left\|_{U} \cdot v_{j}\right\|_{V} \\
& \left(\text { where } b_{j}=\sum_{i \in I_{j}}\left\|z_{i}\right\|_{Z} \cdot u_{i} \text { for each } j \in J_{0}, \text { and we used (12) }\right) \\
\leq & C_{1} C_{2}\left\|\sum_{j \in J_{0}} b_{j}\right\|_{U} \\
\leq & C_{1} C_{2}\left\|\sum_{i=1}^{m}\right\| z_{i}\left\|_{Z_{V}} \cdot u_{i}\right\|_{U}
\end{aligned}
$$

and, thirdly,

$$
\begin{aligned}
\left\|\sum_{j \in J_{0}} \sum_{i \in I_{0}}\right\| & P_{\left(n_{j-1}, n_{j}\right]}^{E}\left(z_{i}\right)\left\|_{Z} \cdot v_{j}\right\|_{V} \\
& \leq\left\|\sum_{j \in J_{0}}\right\| P_{\left(n_{j-1}, n_{j}\right]}^{E}\left(z_{i_{j}^{(1)}}+z_{i_{j}^{(2)}}\right)\left\|_{Z} \cdot v_{j}\right\|_{V}
\end{aligned}
$$

where the $z_{i_{j}}^{(1)}$ 's and $z_{i_{j}}^{(2)}$ 's are chosen as follows: since for every $j \in J_{0}$ the interval $\left(n_{j-1}, n_{j}\right]$ intersects the support of at most two $z_{i}$ 's with $i \in I_{0}$, we can choose, for $j \in J_{0}, i_{j}^{(1)}<i_{j}^{(2)}$ in $\{1,2, \ldots, m\}$ so that $i_{j}^{(2)} \leq i_{j^{\prime}}^{(1)}$ whenever $j<j^{\prime}$ are in $J_{0}$ and so that the above inequality holds. Continuing (18) we have

$$
\begin{aligned}
& \leq\left\|\sum_{j \in J_{0}}\right\| P_{\left(n_{j-1}, n_{j}\right]}^{E}\left(z_{i_{j}^{(1)}}\right)\left\|_{Z} \cdot v_{j}\right\|_{V}+\left\|\sum_{j \in J_{0}}\right\| P_{\left(n_{j-1}, n_{j}\right]}^{E}\left(z_{i_{j}^{(2)}}\right)\left\|_{Z} \cdot v_{j}\right\|_{V} \\
& \leq K C_{2}\left\|\sum_{j \in J_{0}}\right\| z_{i_{j}^{(1)}}\left\|{ }_{Z} \cdot u_{i_{j}^{(1)}}\right\|_{U}+K C_{2}\left\|\sum_{j \in J_{0}}\right\| z_{i_{j}^{(2)}}\left\|_{Z} \cdot u_{i_{j}^{(2)}}\right\|_{U} \\
& \leq 2 K C_{2}\left\|\sum_{i=1}^{m}\right\| z_{i}\left\|_{Z_{V}} \cdot u_{i}\right\|_{U} .
\end{aligned}
$$

Finally, we deduce from (16), (17) and (18) that

$$
\left\|\sum_{j=1}^{k}\right\| P_{\left(n_{j-1}, n_{j}\right]}^{E}(z)\left\|_{Z} \cdot v_{j}\right\|_{V}
$$




$$
\begin{aligned}
& \leq\left\|\sum_{i=1}^{m} \sum_{j \in J_{i}}\right\| P_{\left(n_{j-1}, n_{j}\right]}^{E}\left(z_{i}\right)\left\|_{Z} \cdot v_{j}\right\|_{V} \\
& \quad+\left\|\sum_{j \in J_{0}}\right\| P_{\left(n_{j-1}, n_{j}\right]}^{E}(z)\left\|_{Z} \cdot v_{j}\right\|_{V} \\
& \leq\left\|\sum_{i=1}^{m} \sum_{j \in J_{i}}\right\| P_{\left(n_{j-1}, n_{j}\right]}^{E}\left(z_{i}\right)\left\|_{Z} \cdot v_{j}\right\|_{V} \\
& \quad+\left\|\sum_{j \in J_{0}}\right\| \sum_{i \in I_{j}} z_{i}\left\|_{Z} \cdot v_{j}\right\|_{V} \\
& \quad+\left\|\sum_{j \in J_{0}} \sum_{i \in I_{0}}\right\| P_{\left(n_{j-1}, n_{j}\right]}^{E}\left(z_{i}\right)\left\|_{Z} \cdot v_{j}\right\|_{V} \\
& \leq\left(C_{3}+C_{1} C_{2}+2 K C_{2}\right)\left\|\sum_{i=1}^{m}\right\| z_{i}\left\|_{Z_{V}} \cdot u_{i}\right\|_{U},
\end{aligned}
$$

which finishes the proof of (15).

\section{Embedding Theorems}

In this section we will prove and deduce some consequences of

Theorem 3.1. Assume that $V$ is a Banach space with a normalized and 1-unconditional basis $\left(v_{i}\right)$, and let $X$ be a separable and reflexive space with $V$-lower tree estimates.

a) For every reflexive space $Z$ with an FDD $E=\left(E_{i}\right)$ which contains $X$ there is a blocking $H=\left(H_{i}\right)$ of $\left(E_{i}\right)$ so that $X$ naturally isomorphically embeds into $Z_{V}(H)$.

b) There is a space $\tilde{Y}$ with a shrinking FDD $\tilde{G}=\left(\tilde{G}_{i}\right)$ so that $X$ is a quotient of $\tilde{Y}_{V}(\tilde{G})$.

Corollary 3.2. Assume that $V$ is a reflexive Banach space with a normalized and 1unconditional basis $\left(v_{i}\right)$ satisfying conditions $(W L S)$ and $(S R S)$ as defined in the previous section and having the property that $\left(v_{i}\right)$ is dominated by every normalized block sequence of $\left(v_{i}\right)$. Let $X$ be a separable and reflexive space with $V$-lower tree estimates.

Then $X$ is a subspace of a reflexive space $Z$ with an FDD satisfying $V$-lower estimates and it is a quotient of a reflexive space $Y$ with an FDD satisfying $V$-lower estimates.

Remark. The assumption that $\left(v_{i}\right)$ is dominated by all its normalized block sequences implies that $\left(v_{i}\right)$ satisfies condition $(S R S)$.

Proof. By a theorem of Zippin $\mathrm{Z}$ we can embed $X$ into a reflexive space $W$ with an FDD $E=\left(E_{i}\right)$. Using Theorem 3.1 (a) we can block $\left(E_{i}\right)$ into $F=\left(F_{i}\right)$ so that $X$ embeds into $Z=W_{V}(F)$. Theorem 3.1 (b) provides a space $\tilde{Y}$ with a shrinking FDD $\tilde{G}=\left(\tilde{G}_{i}\right)$ so that $X$ is a quotient of $Y=\tilde{Y}_{V}(\tilde{G})$. By Corollary 2.4 the spaces $Z$ and $Y$ are reflexive.

It follows from Lemma 2.5 that every normalized block tree of $\left(F_{i}\right)$ in $Z$ and of $\left(\tilde{G}_{i}\right)$ in $Y$ has a branch which dominates some normalized block sequence of $\left(v_{i}\right)$ and thus $\left(v_{i}\right)$ itself. It follows that every normalized weakly null tree in $Z$ and in $Y$ has a branch which dominates $\left(v_{i}\right)$, and so, by Proposition 1.2 $Z$ and $Y$ satisfy $V$-lower tree estimates. Finally, by Proposition 1.5 we can find blockings $G=\left(G_{i}\right)$ of $\left(F_{i}\right)$ and $H=\left(H_{i}\right)$ of $\left(\tilde{G}_{i}\right)$ so that $G$ satisfies $V$-lower estimates in $Z$, and $H$ satisfies $V$-lower estimates in $Y$. 
From Corollary 3.2 and Proposition 1.3 we deduce in certain instances the inverse implication of Proposition 1.4.

Corollary 3.3. Assume that $V$ is a reflexive Banach space with a 1-unconditional and normalized basis $\left(v_{i}\right)$ satisfying the conditions of Corollary [3.2.

If $X$ is a reflexive space which satisfies $V$-lower tree estimates, then $X^{*}$ satisfies $V^{*}$-upper tree estimates.

Theorem 3.4. Let $V$ and $U$ be reflexive Banach spaces with normalized, 1-unconditional bases $\left(v_{i}\right)$ and $\left(u_{i}\right)$, respectively, such that $\left(v_{i}\right)$ and $\left(u_{i}^{*}\right)$ both satisfy the conditions of Corollary [3.2. Further assume that every subsequence of $\left(u_{i}\right)$ dominates every normalized block sequence of $\left(v_{i}\right)$ and that every normalized block sequence of $\left(u_{i}\right)$ dominates every subsequence of $\left(v_{i}\right)$.

If $X$ is a separable, reflexive Banach space which satisfies $(V, U)$-tree estimates, then $X$ can be embedded into a reflexive Banach space $Z$ with an $F D D\left(G_{i}\right)$, which satisfies $(V, U)$-estimates in $Z$.

Proof. By Proposition $1.4 X^{*}$ satisfies $U^{*}$-lower tree estimates, and we can apply Corollary 3.2 to deduce that $X^{*}$ is the quotient of a reflexive space $Y^{*}$ with an $\operatorname{FDD}\left(E_{i}^{*}\right)\left(Y^{*}\right.$ being the dual of a space $Y$ with an FDD $\left.\left(E_{i}\right)\right)$ satisfying $U^{*}$-lower estimates in $Y^{*}$. Thus $X$ is a subspace of the reflexive space $Y$ having an FDD $\left(E_{i}\right)$ which, by Proposition 1.3. satisfies $U$-upper estimates in $Y$.

Theorem 3.1part (a) yields a blocking $F=\left(F_{i}\right)$ of $\left(E_{i}\right)$ so that $X$ embeds into $Z=Y_{V}(F)$. As in the proof of Corollary 3.2 we can deduce from the assumptions that $Z$ is reflexive (Corollary [2.4), it satisfies $V$-lower tree estimates (Lemma 2.5] and Proposition [1.2) and that there is a blocking $G=\left(G_{i}\right)$ of $\left(F_{i}\right)$ such that $G$ satisfies $V$-lower estimates in $Z$ (Proposition 1.5).

To complete the proof note that the assumptions of Lemma 2.6 are satisfied, and so the FDD $\left(F_{i}\right)$, and hence also $\left(G_{i}\right)$, satisfies $U$-upper estimates in $Z$.

Remark. Spaces $V$ which satisfy the assumptions of Corollary 3.2 are the $\ell_{p}$ spaces, $1<$ $p<\infty$, and convexified Tsirelson spaces $T_{(p, \gamma)}$ (see [CS] for $1 \leq p<\infty$ and $0<\gamma<1$. In section 4 we will discuss more general versions of these spaces.

The proof of Theorem 3.1 will follow along the lines of the proof of Theorem 1.7 in OS2, where the special case $V=\ell_{p}$, for some $1<p<\infty$, was treated.

From Corollary 4.4 in OS1 we have

Proposition 3.5. Let $X$ be a Banach space which is a subspace of a reflexive space $Z$ with an FDD $A=\left(A_{i}\right)$ having projection constant $K$. Let $\bar{\eta}=\left(\eta_{i}\right) \subset(0,1)$ with $\eta_{i} \downarrow 0$. Then there exist positive integers $N_{1}<N_{2}<\ldots$ such that the following holds. For all $x \in S_{X}$ there exist $x_{i} \in X$ and $t_{i} \in\left(N_{i-1}, N_{i}\right)\left(i \in \mathbb{N}, N_{0}=0\right)$ such that

a) $x=\sum_{i=1}^{\infty} x_{i}$

b) for $i \in \mathbb{N}$ either $\left\|x_{i}\right\|<\eta_{i}$ or $\left\|P_{\left(t_{i-1}, t_{i}\right)}^{A} x_{i}-x_{i}\right\|<\eta_{i}\left\|x_{i}\right\|$,

c) $\left\|P_{\left(t_{i-1}, t_{i}\right)}^{A} x-x_{i}\right\|<\eta_{i}$ for all $i \in \mathbb{N}$,

d) $\left\|x_{i}\right\|<K+1$ for $i \in \mathbb{N}$, and

e) $\left\|P_{t_{i}}^{A} x\right\|<\eta_{i}$ for $i \in \mathbb{N}$.

Moreover, the above conditions hold if $\left(N_{i}\right)$ is replaced by any subsequence of $\left(N_{i}\right)$. 
Parts d) and e) were not explicitly stated in OS1 but follow from the proof.

Proof of Theorem 3.1 part (a). Let $K$ be the projection constant of $E$ in $Z$, and assume that $X$ satisfies $C$ - $V$-lower tree estimates.

Using Proposition [1.6] as in the proof of Proposition [1.5, we find a blocking $\left(F_{i}\right)$ of $\left(E_{i}\right)$ and a sequence $\bar{\delta}=\left(\delta_{i}\right) \subset(0,1), \delta_{i} \downarrow 0$, such that any $\bar{\delta}$-skipped block sequence $\left(\bar{x}_{i}\right) \subset S_{X}$ of any blocking of $\left(F_{i}\right)$ in $Z 2 C$-dominates $\left(v_{i}\right)$ and $\left(v_{i+1}\right)$. We can assume that $\Delta=\sum_{i} \delta_{i}<1$.

It is easy to see that we can block $\left(F_{i}\right)$ into an FDD $G=\left(G_{i}\right)$ so that there exists $\left(e_{n}\right) \subset S_{X}$ with

$$
\left\|e_{n}-P_{n}^{G}\left(e_{n}\right)\right\|_{Z}<\delta_{n} \quad \text { for all } n \in \mathbb{N} .
$$

Finally, we let $N_{1}<N_{2}<\ldots$ be a sequence of positive integers obtained by applying Proposition 3.5 with $\left(A_{i}\right)=\left(G_{i}\right)$ and $\bar{\eta}=\bar{\delta}$.

Now set $H_{i}=\bigoplus_{j=N_{i-1}+1}^{N_{i}} G_{j}$ for $i \in \mathbb{N}$, and let $H=\left(H_{i}\right)$. We consider the space $Z_{V}=Z_{V}(H)$, and claim that $X$ naturally embeds into $Z_{V}$. In order to achieve that we prove that if $x \in S_{X}$, then

$$
\left\|\sum_{i=1}^{\infty}\right\| P_{i}^{H}(x)\left\|_{Z} \cdot v_{i}\right\|_{V} \leq 24 K^{2} C
$$

Since the argument will also work for any further blocking of $\left(H_{i}\right)$ (by the "moreover" part of Proposition [3.5) we obtain for all $x \in S_{X}$

$$
\|x\|_{Z_{V}}=\sup _{0=k_{0}<k_{1}<\ldots<k_{n}}\left\|\sum_{i=1}^{n}\right\| P_{\left(k_{i-1}, k_{i}\right]}^{H}(x)\left\|_{Z} \cdot v_{i}\right\|_{V} \leq 24 K^{2} C .
$$

Let $x \in S_{X}$, and for each $i \in \mathbb{N}$ choose $x_{i} \in X$ and $t_{i} \in\left(N_{i-1}, N_{i}\right)$ so that the properties (a)-(e) of Proposition 3.5 are satisfied with $\left(A_{i}\right)=\left(G_{i}\right)$ and $\bar{\eta}=\bar{\delta}$.

For each $i \in \mathbb{N}$ let $\bar{x}_{i}=\frac{x_{i+1}}{\left\|x_{i+1}\right\|}$ and $\alpha_{i}=\left\|x_{i+1}\right\|$ if $\left\|x_{i+1}\right\| \geq \delta_{i+1}$, and let $\bar{x}_{i}=e_{N_{i}}$ and $\alpha_{i}=0$ if $\left\|x_{i+1}\right\|<\delta_{i+1}$, where $\left(e_{n}\right)$ is a sequence that satisfies (19). Observe that $\left(\bar{x}_{i}\right)$ is a $\bar{\delta}$-skipped block sequence of some blocking of $\left(G_{i}\right)$ (and hence of $\left(F_{i}\right)$ ) and as such it $2 C$-dominates $\left(v_{i}\right)$ and $\left(v_{i+1}\right)$. Using domination of $\left(v_{i}\right)$, we get

$$
\begin{aligned}
\left\|\sum_{i=1}^{\infty} x_{i}\right\|_{Z} & \geq\left\|\sum_{i=1}^{\infty} \alpha_{i} \bar{x}_{i}\right\|-\left\|x_{1}\right\|-\Delta \\
& \geq \frac{1}{2 C}\left\|\sum_{i=1}^{\infty} \alpha_{i} v_{i}\right\|_{V}-(K+1)-\Delta \\
& \geq \frac{1}{2 C}\left\|\sum_{i=1}^{\infty}\right\| x_{i+1}\left\|_{Z} \cdot v_{i}\right\|_{V}-\frac{1}{2 C} \Delta-(K+1)-\Delta
\end{aligned}
$$

and thus

$$
\left\|\sum_{i=1}^{\infty}\right\| x_{i+1}\left\|_{Z} \cdot v_{i}\right\|_{V} \leq 2 C(K+2 \Delta+2) .
$$

Since $\left(\bar{x}_{i}\right)$ also $2 C$-dominates $\left(v_{i+1}\right)$, a similar calculation shows

$$
\left\|\sum_{i=1}^{\infty}\right\| x_{i+1}\left\|_{Z} \cdot v_{i+1}\right\|_{V} \leq 2 C(K+2 \Delta+2) .
$$


Using properties (c) and (e) of Proposition 3.5 with $A=G$ and $\bar{\eta}=\bar{\delta}$, we have

$$
\left\|P_{i}^{H}(x)\right\|_{Z} \leq K\left\|P_{\left(t_{i-1}, t_{i+1}\right)}^{G}(x)\right\|_{Z} \leq K\left(\left\|x_{i}\right\|_{Z}+\left\|x_{i+1}\right\|_{Z}+3 \delta_{i}\right)
$$

for each $i \in \mathbb{N}$. It follows, using (23) and (24), that

$$
\begin{aligned}
\left\|\sum_{i=1}^{\infty}\right\| P_{i}^{H}(x)\left\|_{Z} \cdot v_{i}\right\|_{V} & \leq\left\|P_{1}^{H}(x)\right\|_{Z}+K\left\|\sum_{i=2}^{\infty}\right\| x_{i}\left\|_{Z} \cdot v_{i}\right\|_{V}+K\left\|\sum_{i=2}^{\infty}\right\| x_{i+1}\left\|_{Z} \cdot v_{i}\right\|_{V}+3 K \Delta \\
& \leq 24 K^{2} C .
\end{aligned}
$$

Before we prove part (b) of Theorem 3.1 we need a blocking result due to Johnson and Zippin.

Proposition 3.6. JZ1 Let $T: Y \rightarrow Z$ be a bounded linear operator from a space $Y$ with a shrinking FDD $\left(G_{i}\right)$ into a space $Z$ with an FDD $\left(H_{i}\right)$. Let $\varepsilon_{i} \downarrow 0$. Then there exist blockings $E=\left(E_{i}\right)$ of $\left(G_{i}\right)$ and $F=\left(F_{i}\right)$ of $\left(H_{i}\right)$ so that for all $m<n$ and $y \in S_{\bigoplus_{i \in(m, n)} E_{i}}$ we have $\left\|P_{[1, m)}^{F} T y\right\|<\varepsilon_{m}$ and $\left\|P_{[n, \infty)}^{F} T y\right\|<\varepsilon_{n}$.

Proof of Theorem 3.1 part (b). By Lemma 3.1 in OS1 we can, after renorming $X$ if necessary, regard $X^{*}$ (isometrically) as a subspace of a reflexive space $Y^{*}$ (being the dual of a reflexive space $Y$ ) with bimonotone $\operatorname{FDD}\left(E_{i}^{*}\right)$ such that $c_{00}\left(\oplus_{i=1}^{\infty} E_{i}^{*}\right) \cap X^{*}$ is dense in $X^{*}$. We have a natural quotient map $Q: Y \rightarrow X$. By a Theorem of Zippin [Z] we may regard $X$ (isometrically) as a subspace of a reflexive space $Z$ with an FDD $\left(F_{i}\right)$. Let $K$ be the projection constant of $\left(F_{i}\right)$ in $Z$, and choose $C>0$ such that $X$ satisfies $C$ - $V$-lower tree estimates.

Using Proposition [1.6 as in the proof of Proposition [1.5 we find a sequence $\bar{\delta}=\left(\delta_{i}\right) \subset$ $(0,1), \delta_{i} \downarrow 0$, so that if $\left(x_{i}\right) \subset X$ is any $\bar{\delta}$-skipped block sequence of any blocking of $\left(F_{i}\right)$, then $\left(x_{i}\right) 2 C$-dominates $\left(v_{i}\right)$, and moreover, using standard perturbation arguments and making $\bar{\delta}$ smaller if necessary, we can assume that if $\left(z_{i}\right) \subset Z$ satisfies $\left\|x_{i}-z_{i}\right\|<\delta_{i}$ for all $i \in \mathbb{N}$, then $\left(z_{i}\right)$ is a basic sequence equivalent to $\left(x_{i}\right)$ with projection constant at most $2 K$. We also require that

$$
\Delta=\sum_{i=1}^{\infty} \delta_{i}<\frac{1}{7}
$$

Choose a sequence $\bar{\varepsilon}=\left(\varepsilon_{i}\right) \subset(0,1)$ with $\varepsilon_{i} \downarrow 0$ and

$$
3 K(K+1) \sum_{j=i}^{\infty} \varepsilon_{j}<\delta_{i}^{2} \quad \text { for all } i \in \mathbb{N}
$$

After blocking $\left(F_{i}\right)$ if necessary, we can assume that for any subsequent blocking $D$ of $F$ there is a sequence $\left(e_{i}\right)$ in $S_{X}$ such that

$$
\left\|e_{i}-P_{i}^{D}\left(e_{i}\right)\right\|_{Z}<\varepsilon_{i} / 2 K \quad \text { for all } i \in \mathbb{N} .
$$

By Proposition 3.6 we may assume, after further blocking our FDDs if necessary, that

$$
\begin{aligned}
& \text { for all } m<n \text { and } y \in S_{\oplus_{i \in(m, n)} E_{i} \text { we have }} \\
& \qquad\left\|P_{[1, m)}^{F} \circ Q(y)\right\|<\varepsilon_{m} \quad \text { and }\left\|P_{[n, \infty)}^{F} \circ Q(y)\right\|<\varepsilon_{n},
\end{aligned}
$$


and moreover the same holds if one passes to any blocking of $\left(E_{i}\right)$ and the corresponding blocking of $\left(F_{i}\right)$.

For $i \in \mathbb{N}$ let $\tilde{E}_{i}$ be the quotient space of $E_{i}$ determined by $Q$, i.e. if $y \in E_{i}$, then the norm of $\tilde{y}$, the equivalence class of $y$ in $E_{i}$, is given by $\|\tilde{y}\|=\|Q(y)\|$. Passing to a further blocking of $\left(E_{i}\right)$ (and the corresponding blocking of $\left(F_{i}\right)$ ), we may assume that $\tilde{E}_{i} \neq\{0\}$ for $i \in \mathbb{N}$. Given $y=\sum y_{i} \in \mathrm{c}_{00}\left(\oplus_{i=1}^{\infty} E_{i}\right), y_{i} \in E_{i}$ for $i \in \mathbb{N}$, we set $\tilde{y}=\sum \tilde{y}_{i} \in \mathrm{c}_{00}\left(\oplus_{i=1}^{\infty} \tilde{E}_{i}\right)$ and

$$
\|\tilde{y}\|=\max _{m \leq n}\left\|\sum_{i=m}^{n} Q\left(y_{i}\right)\right\|=\max _{m \leq n}\left\|Q \circ P_{[m, n]}^{E}(y)\right\| .
$$

We let $\tilde{Y}$ be the completion of $\mathrm{c}_{00}\left(\oplus_{i=1}^{\infty} \tilde{E}_{i}\right)$ with respect to $\|\cdot\|$. Since $\left(E_{i}\right)$ is a bimonotone FDD in $Y$, we have $\|\tilde{y}\| \leq\|y\|$ for all $y \in \mathrm{c}_{00}\left(\bigoplus_{i=1}^{\infty} E_{i}\right)$, and hence the map $y \mapsto \tilde{y}$ extends to a norm one map from $Y$ to $\tilde{Y}$. By the definition of $\|\cdot\|$ we have $\|Q y\| \leq\|\tilde{y}\|$ for any $y \in \mathrm{c}_{00}\left(\oplus_{i=1}^{\infty} E_{i}\right)$. It follows that $\tilde{y} \rightarrow Q(y)$ extends to a norm one map $\tilde{Q}: \tilde{Y} \rightarrow X$ with $\tilde{Q}(\tilde{y})=Q(y)$ for all $y \in Y$.

In order to continue our proof we will need the following proposition from OS2].

Proposition 3.7. OS2, Proposition 2.6]

a) $\left(\tilde{E}_{i}\right)$ is a bimonotone, shrinking FDD for $\tilde{Y}$.

b) $\tilde{Q}$ is a quotient map from $\tilde{Y}$ onto $X$. More precisely if $x \in X$ and $y \in Y$ is such that $Q(y)=x,\|y\|=\|x\|$ and $y=\sum y_{i}$ with $y_{i} \in E_{i}$ for all $i \in \mathbb{N}$, then $\tilde{y}=\sum \tilde{y}_{i} \in \tilde{Y}$, $\|\tilde{y}\|=\|y\|$ and $\tilde{Q}(\tilde{y})=x$.

c) Let $\left(\tilde{y}_{i}\right)$ be a block sequence of $\left(\tilde{E}_{i}\right)$ in $B_{\tilde{Y}}$, and assume that $\left(\tilde{Q}\left(\tilde{y}_{i}\right)\right)$ is a basic sequence with projection constant $\bar{K}$ and $a=\inf _{i}\left\|\tilde{Q}\left(\tilde{y}_{i}\right)\right\|>0$. Then for all $\left(a_{i}\right) \in$ $\mathrm{c}_{00}$ we have

$$
\left\|\sum a_{i} \tilde{Q}\left(\tilde{y}_{i}\right)\right\| \leq\left\|\sum a_{i} \tilde{y}_{i}\right\| \leq \frac{3 \bar{K}}{a}\left\|\sum a_{i} \tilde{Q}\left(\tilde{y}_{i}\right)\right\| .
$$

To finish the proof of Theorem 3.1 (b) it suffices to find a constant $L<\infty$ and a blocking $\tilde{G}=\left(\tilde{G}_{i}\right)$ of $\left(\tilde{E}_{i}\right)$ with the following property. For any $x \in S_{X}$ there exists a $\tilde{y}=\sum \tilde{y}_{i} \in \tilde{Y}$, $\tilde{y}_{i} \in \tilde{G}_{i}$ for $i \in \mathbb{N}$, so that

$$
\begin{aligned}
& \|\tilde{Q}(\tilde{y})-x\|<1 / 2, \\
& \left\|\sum_{j=1}^{k}\right\| P_{\left(n_{j-1}, n_{j}\right]}^{\tilde{G}}(\tilde{y})\left\|\cdot v_{j}\right\|_{V} \leq L \\
& \text { for any choice of } k \text { and } n_{1}<n_{2}<\ldots<n_{k} \text { in } \mathbb{N}\left(n_{0}=0\right) .
\end{aligned}
$$

Once this is accomplished, we consider the space $\tilde{Y}_{V}=\tilde{Y}_{V}(\tilde{G})$. Given $x=x_{0} \in S_{X}$, the property of $\tilde{G}$ allows us to recursively choose $x_{n} \in \frac{1}{2^{n}} B_{X}$ and $\tilde{y}_{n} \in \frac{L}{2^{n-1}} B_{\tilde{Y}_{V}}, n \in \mathbb{N}$, so that $x_{n}=x_{n-1}-\tilde{Q}\left(\tilde{y}_{n}\right)$ for all $n \in \mathbb{N}$. It follows that $\sum_{n=1}^{\infty} \tilde{y}_{n}$ converges in $\tilde{Y}_{V}$ with $\left\|\sum_{n=1}^{\infty} \tilde{y}_{n}\right\| \leq 2 L$ and $\tilde{Q}\left(\sum_{n=1}^{\infty} \tilde{y}_{n}\right)=x$. Thus $\tilde{Q}: \tilde{Y}_{V} \rightarrow X$ remains surjective, which finishes the proof.

In order to show the existence of a suitable blocking $\tilde{G}$ of $\tilde{E}$ we need the following result from OS2. 
Lemma 3.8. [S2, Lemma 2.7] Assume that (28) holds for our original map $Q: Y \rightarrow X$. Then there exist integers $0=N_{0}<N_{1}<\ldots$ so that if for each $i \in \mathbb{N}$ we define

$$
\begin{aligned}
& C_{i}=\bigoplus_{j=N_{i-1}+1}^{N_{i}} E_{j}, \quad D_{i}=\bigoplus_{j=N_{i-1}+1}^{N_{i}} F_{j}, \\
& L_{i}=\left\{j \in \mathbb{N}: N_{i-1}<j \leq \frac{N_{i-1}+N_{i}}{2}\right\}, \\
& R_{i}=\left\{j \in \mathbb{N}: \frac{N_{i-1}+N_{i}}{2}<j \leq N_{i}\right\}, \\
& C_{i, L}=\bigoplus_{j \in L_{i}} E_{j} \quad \text { and } \quad C_{i, R}=\bigoplus_{j \in R_{i}} E_{j},
\end{aligned}
$$

then the following holds. Let $x \in S_{X}, 0 \leq m<n$ and $\varepsilon>0$, and assume that $\| x-$ $P_{(m, n)}^{D}(x) \|<\varepsilon$. Then there exists $y \in B_{Y}$ with $y \in C_{m, R} \oplus\left(\bigoplus_{i \in(m, n)} C_{i}\right) \oplus C_{n, L}$ (where $C_{0, R}=\{0\}$ ) and $\|Q y-x\|<K\left[2 \varepsilon+\varepsilon_{m+1}\right]$ (recall that $K$ is the projection constant of $\left(F_{i}\right)$ in $Z$ ).

Let $\left(C_{i}\right)$ and $\left(D_{i}\right)$ be the blockings given by Lemma 3.8 We now apply Proposition 3.5 with $\left(A_{i}\right)=\left(D_{i}\right)$ and $\bar{\eta}=\bar{\varepsilon}$ to obtain a sequence $N_{1}<N_{2}<\ldots$ in $\mathbb{N}$ so that the conclusions of the proposition are satisfied. We now come to our final blockings: for each $i \in \mathbb{N}$ set $G_{i}=\oplus_{j=N_{i-1}+1}^{N_{i}} C_{j}$ and let $H_{i}=\oplus_{j=N_{i-1}+1}^{N_{i}} D_{j}\left(N_{0}=0\right)$. Put $G=\left(G_{i}\right)$ and $H=\left(H_{i}\right)$. Let $\tilde{G}=\left(\tilde{G}_{i}\right)$ be the corresponding blocking of $\left(\tilde{E}_{i}\right)$.

Fix a sequence $\left(e_{i}\right)$ in $S_{X}$ so that (27) holds. Let $x \in S_{X}$. By the choice of $N_{1}, N_{2}, \ldots$, for each $i \in \mathbb{N}$, there are $x_{i} \in(K+1) B_{X}$ and $t_{i} \in\left(N_{i-1}, N_{i}\right)$ such that $x=\sum_{i=1}^{\infty} x_{i}$ and for all $i \in \mathbb{N}$ either $\left\|x_{i}\right\|<\varepsilon_{i}$ or $\left\|P_{\left(t_{i-1}, t_{i}\right)}^{D} x_{i}-x_{i}\right\|<\varepsilon_{i}\left\|x_{i}\right\|\left(t_{0}=0\right)$. For each $i \in \mathbb{N}$ let $\bar{x}_{i}=\frac{x_{i+1}}{\left\|x_{i+1}\right\|}$ and $\alpha_{i}=\left\|x_{i+1}\right\|$ if $\left\|x_{i+1}\right\| \geq \varepsilon_{i+1}$, and let $\bar{x}_{i}=e_{N_{i}}$ and $\alpha_{i}=0$ if $\left\|x_{i+1}\right\|<\varepsilon_{i+1}$. Since

$$
\left\|\bar{x}_{i}-P_{\left(t_{i}, t_{i+1}\right)}^{D}\left(\bar{x}_{i}\right)\right\|<\varepsilon_{i+1} \quad \text { for all } i \in \mathbb{N},
$$

there exists $\left(y_{i}\right) \subset B_{Y}$ with $y_{i} \in C_{t_{i}, R} \oplus\left(\bigoplus_{j \in\left(t_{i}, t_{i+1}\right)} C_{j}\right) \oplus C_{t_{i+1}, L}$ and

$$
\left\|Q\left(y_{i}\right)-\bar{x}_{i}\right\|<3 K \varepsilon_{i+1}, \quad i \in \mathbb{N} .
$$

Also, if $\left\|x_{1}\right\|<\varepsilon_{1}$, then set $y_{0}=0$, and if $\left\|x_{1}\right\| \geq \varepsilon_{1}$, then choose $y_{0} \in(K+1) B_{Y}$ such that $y_{0} \in\left(\bigoplus_{j \in\left(0, t_{1}\right)} C_{j}\right) \oplus C_{t_{1}, L} \subset G_{1}$ and $\left\|Q\left(y_{0}\right)-x_{1}\right\|<3 K(K+1) \varepsilon_{1}$.

Set $\bar{x}=x_{1}+\sum_{i=1}^{\infty} \alpha_{i} \bar{x}_{i}$, and note that (this series converges and) by (25) and (26)

$$
\|x-\bar{x}\| \leq \sum_{i=2}^{\infty} \varepsilon_{i}<\frac{1}{4} .
$$

As a $\bar{\delta}$-skipped block sequence of a blocking of $\left(F_{i}\right)$ (this follows from (31) and (26)), $\left(\bar{x}_{i}\right)$ is a basic sequence that $2 C$-dominates $\left(v_{i}\right)$. Since, by (32), $\left\|\tilde{Q}\left(\tilde{y}_{i}\right)-\bar{x}_{i}\right\|<3 K \varepsilon_{i+1}<\delta_{i}$ for all $i \in \mathbb{N}$, the sequence $\left(\tilde{Q}\left(\tilde{y}_{i}\right)\right)$ is also a basic sequence equivalent to $\left(\bar{x}_{i}\right)$ with projection constant at most $2 K$. Furthermore, we have $\inf _{i}\left\|\tilde{Q}\left(\tilde{y}_{i}\right)\right\| \geq \inf _{i}\left(\left\|\bar{x}_{i}\right\|-\delta_{i}\right)>6 / 7$, and thus, by Proposition 3.7 (c),

$$
\left\|\sum a_{i} \tilde{Q}\left(\tilde{y}_{i}\right)\right\| \leq\left\|\sum a_{i} \tilde{y}_{i}\right\| \leq 7 K\left\|\sum a_{i} \tilde{Q}\left(\tilde{y}_{i}\right)\right\| \quad \text { for all }\left(a_{i}\right) \in \mathrm{c}_{00} .
$$


Thus $\left(\tilde{y}_{i}\right)$ is a basic sequence equivalent to $\left(\bar{x}_{i}\right)$ and, in particular, $\sum_{i=1}^{\infty} \alpha_{i} \tilde{y}_{i}$ converges. Putting $\tilde{y}=\tilde{y}_{0}+\sum_{i=1}^{\infty} \alpha_{i} \tilde{y}_{i}$ we have

$$
\begin{aligned}
\|\tilde{Q} \tilde{y}-\bar{x}\| & \leq\left\|\tilde{Q} \tilde{y}_{0}-x_{1}\right\|+\sum_{i=1}^{\infty}\left|\alpha_{i}\right|\left\|\tilde{Q} \tilde{y}_{i}-\bar{x}_{i}\right\| \\
& \leq 3 K(K+1) \sum_{i=1}^{\infty} \varepsilon_{i}<1 / 4
\end{aligned}
$$

and hence, by (33), $\|\tilde{Q} \tilde{y}-x\|<1 / 2$, so we have (29).

We now fix integers $k$ and $0=n_{0}<n_{1}<\cdots<n_{k}$. For any $i \in \mathbb{N}$ we have $P_{i}^{\tilde{G}}(\tilde{y})=$ $P_{i}^{\tilde{G}}\left(\tilde{y}_{i-1}+\tilde{y}_{i}\right)$. It follows that

$$
\begin{aligned}
&\left\|\sum_{s=1}^{k}\right\| P_{\left(n_{s-1}, n_{s}\right]}^{\tilde{G}}(\tilde{y})\left\|\cdot v_{s}\right\|_{V} \leq\left\|\tilde{y}_{0}\right\|+\left\|\sum_{s=1}^{k}\right\| \sum_{\substack{i=n_{s-1}+1 \\
i \geq 2}}^{n_{s}} \alpha_{i-1} \tilde{y}_{i-1} \| \\
&+\left\|\sum_{s}\right\|_{V}\left\|\sum_{i=n_{s-1}+1}^{n_{s}} \alpha_{i} \tilde{y}_{i}\right\|\left\|v_{s}\right\|_{V} .
\end{aligned}
$$

We now show how to bound the third term of the right-hand side of the above inequality. A similar argument will give an estimate for the second term, and then (30) will follow with $L=142 K^{2} C$.

For each $s=1, \ldots, k$ let $\tilde{w}_{s}=\sum_{i=n_{s-1}+1}^{n_{s}} \alpha_{i} \tilde{y}_{i}$ and $b_{s}=\sum_{i=n_{s-1}+1}^{n_{s}} \alpha_{i} \bar{x}_{i}$. Note that by (31) and (26)

$$
\begin{aligned}
\left\|P_{\left(t_{n_{s-1}+1}, t_{n_{s}+1}\right)}^{D}\left(b_{s}\right)-b_{s}\right\| & \leq \sum_{i=n_{s-1}+1}^{n_{s}}\left|\alpha_{i}\right| \cdot 2 K \cdot\left\|P_{\left(t_{i}, t_{i+1}\right)}^{D} \bar{x}_{i}-\bar{x}_{i}\right\| \\
& <2 K(K+1) \sum_{i=n_{s-1}+1}^{n_{s}} \varepsilon_{i+1}<\delta_{s}^{2} \quad(s=1, \ldots, k) .
\end{aligned}
$$

For $s>k$ set $n_{s}=n_{k}+(s-k)$. If $s>k$ or $\left\|b_{s}\right\|<\delta_{s}$, then we let $\bar{b}_{s}=\bar{x}_{n_{s}}$ and $\beta_{s}=0$. If $s \leq k$ and $\left\|b_{s}\right\| \geq \delta_{s}$, then we let $\bar{b}_{s}=\frac{b_{s}}{\left\|b_{s}\right\|}$ and $\beta_{s}=\left\|b_{s}\right\|$.

It follows from (36) and (31) that $\left(\bar{b}_{s}\right)$ is a $\bar{\delta}$-skipped block sequence of some blocking of $\left(F_{i}\right)$, and hence it is a basic sequence that $2 C$-dominates $\left(v_{i}\right)$. From (32) and (34) we have

$$
\begin{aligned}
\left\|\tilde{Q}\left(\tilde{w}_{s}\right)-b_{s}\right\| & \leq \sum_{i=n_{s-1}+1}^{n_{s}}\left|\alpha_{i}\right|\left\|\tilde{Q}\left(\tilde{y}_{i}\right)-\bar{x}_{i}\right\| \\
& <3 K(K+1) \sum_{i=n_{s-1}+1}^{n_{s}} \varepsilon_{i+1}<\delta_{s}
\end{aligned}
$$

and

$$
\left\|\tilde{w}_{s}\right\| \leq 7 K\left\|\tilde{Q}\left(\tilde{w}_{s}\right)\right\| \quad(s=1, \ldots, k) .
$$


Putting $\gamma_{s}=\beta_{s}$ for $s=1, \ldots, k$ and $\gamma_{s}=\alpha_{n_{s}}$ for $s>k$, we obtain the following.

$$
\begin{aligned}
\left\|\sum_{s=1}^{k}\right\| \tilde{w}_{s}\left\|\cdot v_{s}\right\|_{V} & \leq 7 K\left\|\sum_{s=1}^{k}\right\| \tilde{Q}\left(\tilde{w}_{s}\right)\left\|\cdot v_{s}\right\|_{V} \\
& \leq 7 K\left\|\sum_{s=1}^{k}\right\| b_{s}\left\|\cdot v_{s}\right\|_{V}+7 K \Delta \\
& \leq 7 K\left\|\sum_{s=1}^{\infty} \gamma_{s} v_{s}\right\|_{V}+14 K \Delta \quad \text { (from (138)) } \\
& \leq 7 K \cdot 2 C\left\|\sum_{s=1}^{\infty} \gamma_{s} \bar{b}_{s}\right\|+14 K \Delta \quad \text { (as }\left(v_{s}\right) \text { is 1-unconditional) } \\
& \leq 14 K C\left\|\sum_{i=1}^{\infty} \alpha_{i} \bar{x}_{i}\right\|+14 K C \Delta+14 K \Delta \\
& \leq 14 K C \cdot(K+3)+14 K C \Delta+14 K \Delta<70 K^{2} C,
\end{aligned}
$$

where the last line follows from (33) and from $\left\|x_{1}\right\| \leq K+1$. An almost identical calculation gives the same esimate for the second term in (35), and hence we obtain (30) with $L=$ $142 K^{2} C$. This completes the proof of part (b) of Theorem 3.1

\section{Asymptotic estimates}

Throughout this section we assume that $U$ and $V$ are Banach spaces with normalized, 1-subsymmetric bases $\left(u_{i}\right)$ and $\left(v_{i}\right)$, respectively, i.e. $\left(u_{i}\right)$ and $\left(v_{i}\right)$ are 1-unconditional and 1-spreading $\left(\left\|\sum a_{i} v_{i}\right\|=\left\|\sum a_{i} v_{n_{i}}\right\|\right.$ whenever $\left(a_{i}\right) \in \mathrm{c}_{00}$ and $\left.n_{1}<n_{2}<\ldots\right)$.

Definition. Let $Z$ be a Banach space with an $\operatorname{FDD}\left(E_{i}\right)$, and let $C \geq 1$. We say that $\left(E_{i}\right)$ satisfies asymptotic $C$-V-lower estimates (in $Z$ ) if for all $n \in \mathbb{N}$ we have:

$$
\left\|\sum_{i=1}^{n} x_{i}\right\| \geq C^{-1}\left\|\sum_{i=1}^{n}\right\| x_{i}\left\|v_{i}\right\| \quad \text { whenever }\left(x_{i}\right)_{i=1}^{n} \text { is a block sequence of }\left(E_{i}\right)_{i=n}^{\infty} \text {. }
$$

We say that $\left(E_{i}\right)$ satisfies asymptotic $C$-U-upper estimates (in $Z$ ) if for all $n \in \mathbb{N}$ we have:

$$
\left\|\sum_{i=1}^{n} x_{i}\right\| \leq C\left\|\sum_{i=1}^{n}\right\| x_{i}\left\|u_{i}\right\| \quad \text { whenever }\left(x_{i}\right)_{i=1}^{n} \text { is a block sequence of }\left(E_{i}\right)_{i=n}^{\infty} \text {. }
$$

We say that $\left(E_{i}\right)$ satisfies asymptotic $C-(V, U)$-estimates (in $\left.Z\right)$ if it satisfies asymptotic $C$ - $V$-lower and asymptotic $C$ - $U$-upper estimates in $Z$.

We say that $\left(E_{i}\right)$ satisfies asymptotic $V$-lower estimates, $U$-upper estimates or $(V, U)$ estimates (in $Z$ ) if there is a $C \geq 1$ so that $\left(E_{i}\right)$ satisfies asymptotic $C$ - $V$-lower estimates, $C$ - $U$-upper estimates or $C$ - $(V, U)$-estimates in $Z$, respectively.

As before we also introduce the coordinate-free version of asymptotic lower and upper estimates, which can also be found (defined in more general situations) in [MT] and [MMT].

Definition. We say that a reflexive space $X$ satisfies asymptotic $C$-V-lower tree estimates or asymptotic $C$-U-upper tree estimates if, for every $k$, every normalized weakly null tree of length $k$ in $X$ has a branch which $C$-dominates $\left(v_{i}\right)_{i=1}^{k}$ or is $C$-dominated by $\left(u_{i}\right)_{i=1}^{k}$, 
respectively. We say $X$ satisfies asymptotic $C-(V, U)$-tree estimates if it satisfies asymptotic $C$ - $V$-lower tree estimates and asymptotic $C$ - $U$-upper tree estimates.

We will say that $X$ satisfies asymptotic $V$-lower tree, $U$-upper tree or $(V, U)$-tree estimates if there is a $C \geq 1$ so that $X$ satisfies asymptotic $C$ - $V$-lower tree, $C$ - $U$-upper tree or $C$ $(V, U)$-tree estimates, respectively.

The following dualities can be shown as in Propositions 1.3 and 1.4

Proposition 4.1. Assume that $V$ is a space with a normalized, 1-subsymmetric basis $\left(v_{i}\right)$.

a) For a space $Z$ with an FDD $\left(E_{i}\right)$ the following statements are equivalent.

1) $\left(E_{i}\right)$ satisfies asymptotic $V$-lower estimates in $Z$.

2) $\left(E_{i}^{*}\right)$ satisfies asymptotic $V^{(*)}$-upper estimates in $Z^{(*)}$.

b) If $X$ is a reflexive space which satisfies asymptotic $C$ - $V$-upper tree estimates for some $C \geq 1$, then, for any $\varepsilon>0, X^{*}$ satisfies asymptotic $(2 C+\varepsilon)-V^{(*)}$-lower tree estimates.

For a space $V$ with a normalized, 1-subsymmetric basis $\left(v_{i}\right)$ and for $0<\gamma<1$ we will introduce the Tsirelson space $T(V, \gamma)$ associated to $V$ and $\gamma$ as follows. It is the space defined as the completion of $c_{00}$ under the norm $\|\cdot\|_{T(V, \gamma)}$, where

$$
\|x\|_{T(V, \gamma)}=\max _{\ell \in \mathbb{N}_{0}}\|x\|_{\ell, T(V, \gamma)} \quad \text { for all } x \in \mathrm{c}_{00},
$$

and the norms $\|\cdot\|_{\ell, T(V, \gamma)}, \ell \in \mathbb{N}$, on $\mathrm{c}_{00}$ are defined recursively as follows. For $x=\left(x_{i}\right) \in \mathrm{c}_{00}$ we put

$$
\|x\|_{0, T(V, \gamma)}=\|x\|_{\infty}=\max _{i \in \mathbb{N}}\left|x_{i}\right|
$$

and, assuming $\|\cdot\|_{\ell, T(V, \gamma)}$ has been defined, we put

$$
\|x\|_{\ell+1, T(V, \gamma)}=\|x\|_{\ell, T(V, \gamma)} \vee \max _{n \in \mathbb{N}, n \leq A_{1}<A_{2}<\cdots<A_{n}} \gamma\left\|\sum_{i=1}^{n}\right\| P_{A_{i}}(x)\left\|_{\ell, T(V, \gamma)} \cdot v_{i}\right\|_{V},
$$

where for $A, B \subset \mathbb{N}$ and $n \in \mathbb{N}, n \leq A$ means that $n \leq a$ for all $a \in A$, and $A<B$ means that $a<b$ for all $a \in A$ and $b \in B$. $P_{A}$, for $A \subset \mathbb{N}$, denotes the projection $\sum a_{i} e_{i} \mapsto \sum_{i \in A} a_{i} e_{i}$ on $\mathrm{c}_{00}$.

As in the case of $V=\ell_{1}$, which yields the standard Tsirelson space (cf. [CS]), it is easy to see that $\|\cdot\|_{T(V, \gamma)}$ satisfies the following implicit equation.

$$
\|x\|_{T(V, \gamma)}=\|x\|_{\infty} \vee \sup _{n \geq 2, n \leq A_{1}<A_{2}<\cdots<A_{n}} \gamma\left\|\sum_{i=1}^{n}\right\| P_{A_{i}}(x)\left\|_{T(V, \gamma)} \cdot v_{i}\right\|_{V}
$$

whenever $x \in T(V, \gamma)$.

Proposition 4.2. Let $V$ be a Banach space with a normalized, 1-subsymmetric basis $\left(v_{i}\right)$. Let $X$ be a Banach space with a normalized basis $\left(e_{i}\right)$ satisfying asymptotic $C$-V -lower estimates for some $C \geq 1$, i.e.

$$
\left\|\sum_{i=1}^{n} a_{i} x_{i}\right\| \geq C^{-1}\left\|\sum_{i=1}^{n} a_{i} v_{i}\right\|_{V}
$$

whenever $n \in \mathbb{N},\left(x_{i}\right)_{i=1}^{n}$ is a normalized block sequence of $\left(e_{i}\right)_{i=n}^{\infty}$ in $X$ and $\left(a_{i}\right)_{i=1}^{n} \subset \mathbb{R}$. Let $K$ be the projection constant of $\left(e_{i}\right)$ in $X$. Then $\left(e_{i}\right) K$-dominates the unit vector basis $\left(t_{i}\right)$ of $T(V, \gamma)$ whenever $0<\gamma<1$ and $\gamma \leq(K C)^{-1}$. 
Proof. There is an equivalent norm $\|\cdot\|$ on $X$ with respect to which $\left(e_{i}\right)$ is bimonotone and which satisfies $\|x\| \leq\|x\| \leq K\|x\|$ for all $x \in X$. In $\|\cdot\|$ the basis $\left(e_{i}\right)$ satisfies asymptotic $(K C)$ - $V$-lower estimates. We can easily show by induction on $l \in \mathbb{N}_{0}$ that

$$
\left\|\mid \sum a_{i} e_{i}\right\| \geq\left\|\sum a_{i} t_{i}\right\|_{\ell, T(V, \gamma)} \quad \text { whenever }\left(a_{i}\right) \in \mathrm{c}_{00}
$$

which proves the proposition.

Proposition 4.3. Let $V$ be a Banach space with a normalized, 1-subsymmetric basis $\left(v_{i}\right)$, and let $0<\gamma<1$.

a) The unit vector basis $\left(t_{i}\right)$ in $T(V, \gamma)$ is a normalized, 1-unconditional basis of $T(V, \gamma)$.

b) If $\left(v_{i}\right)$ is not equivalent to the unit vector basis of $\mathrm{c}_{0}$ (i.e. $\left\|\sum_{i=1}^{n} v_{i}\right\| \rightarrow \infty$ as $n \rightarrow \infty)$, then $T(V, \gamma)$ is reflexive.

c) $\left(t_{i}\right)$ is 1-dominated by every normalized block sequence of $\left(t_{i}\right)$ and, in particular (as subsequences are normalized block sequences), $T(V, \gamma)$ satisfies $(S R S)$ with $c=1$.

d) If $\gamma<1 / 4$, then $T(V, \gamma)$ satisfies (WLS) for any $d<1$.

Proof. (a) is trivial and (b) follows from the fact that $T(V, \gamma)$ does not contain $\ell_{1}$ or $\mathrm{c}_{0}$, which can be shown as in the case $V=\ell_{1}$ (cf. [CS]), and from the theorem of James (cf. [LT]) that states that a space with an unconditional basis is reflexive if and only if it does not contain copies of $c_{0}$ or $\ell_{1}$.

For a normalized block sequence $\left(x_{i}\right)$ of $\left(t_{i}\right)$ it follows from (39) that

$$
\left\|\sum a_{i} x_{i}\right\| \geq \max _{n, n \leq k_{0}<k_{1}<\cdots<k_{n}} \gamma\left\|\sum_{j=1}^{n}\right\| \sum_{i=k_{j-1}}^{k_{j}-1} a_{i} x_{i}\left\|\cdot v_{j}\right\|_{V}
$$

whenever $\left(a_{i}\right) \in \mathrm{c}_{00}$. Thus $\left(x_{i}\right)$ is a normalized, bimonotone basic sequence satisfying asymptotic $C$ - $V$-lower estimates for $C=\gamma^{-1}$, and hence (c) follows from Proposition 4.2.

Claim (d) follows from the following lemma.

Lemma 4.4. For $m \in \mathbb{N}$, let $S^{m}: \mathrm{c}_{00} \rightarrow \mathrm{c}_{00}$ be the backward shift by $m$ coordinates, i.e.

$$
S^{m}\left(\sum_{i=1}^{\infty} a_{i} e_{i}\right)=\sum_{i=1}^{\infty} a_{i+m} e_{i} \quad \text { for all }\left(a_{i}\right) \in \mathrm{c}_{00} .
$$

Assume that $0<\gamma<1 / 4$ and that $m<n$ in $\mathbb{N}$ satisfy

$$
\frac{m}{n-m} \cdot \frac{1}{1-4 \gamma}<1
$$

Then for any $x \in \mathrm{c}_{00}$ with $n \leq \min \operatorname{supp}(x)$ we have

$$
\left\|S^{m}(x)\right\|_{T(V, \gamma)} \geq\left(1-\frac{m}{n-m} \cdot \frac{1}{1-4 \gamma}\right)\|x\|_{T(V, \gamma)} .
$$

Proof. Set $\|\cdot\|=\|\cdot\|_{T(V, \gamma)}$ and $\|\cdot\|_{\ell}=\|\cdot\|_{\ell, T(V, \gamma)}$ for $\ell \in \mathbb{N}$. Fix $m \in \mathbb{N}$. Given $\ell, n \in \mathbb{N}$ with $m<n$ and $\frac{m}{n-m} \cdot \frac{1}{1-4 \gamma}<1$, we put

$$
\Delta(0, n)=0 \quad \text { and } \quad \Delta(\ell, n)=\frac{m}{n-m} \sum_{i=0}^{\ell-1}(4 \gamma)^{i} .
$$


We will show by induction on $\ell \in \mathbb{N}_{0}$ that

$$
\begin{aligned}
& \left\|S^{m}(x)\right\|_{\ell} \geq(1-\Delta(\ell, n)) \cdot\|x\|_{\ell} \\
& \quad \text { whenever } x \in \mathrm{c}_{00}, m<n \leq \min \operatorname{supp}(x) \text { and } \frac{m}{n-m} \cdot \frac{1}{1-4 \gamma}<1 .
\end{aligned}
$$

Clearly, (41) yields the lemma, and the inequality is trivially true for $\ell=0$. Assume that (41) is true for some $\ell \in \mathbb{N}_{0}$. Fix $n \in \mathbb{N}$ and $x \in \mathrm{c}_{00}$ such that $m<n \leq \min \operatorname{supp}(x)$, $\frac{m}{n-m} \cdot \frac{1}{1-4 \gamma}<1$ and $\|x\|_{\ell+1}=1$.

If $\|x\|_{\ell+1}=\|x\|_{\ell}$, then (41) follows for $\ell+1$ from the induction hypothesis. Otherwise we can find $k \in \mathbb{N}, k \geq n$, and sets $k \leq A_{1}<A_{2}<\cdots<A_{k}$ so that

$$
\|x\|_{\ell+1}=\gamma\left\|\sum_{i=1}^{k}\right\| P_{A_{i}}(x)\left\|_{\ell} \cdot v_{i}\right\|_{V}=1
$$

with $\left\|P_{A_{i}}(x)\right\|_{\ell} \leq 1$ for $i=1,2, \ldots, k$ (otherwise $\|x\|_{\ell} \geq\left\|P_{A_{i}}(x)\right\|_{\ell}>1=\|x\|_{\ell+1}$ ).

Let $I \subset\{1,2, \ldots, k\}$, \#I=k-m, so that

$$
\left\|\sum_{i \in I}\right\| P_{A_{i}}(x)\left\|_{\ell} \cdot v_{i}\right\|_{V}=\underset{\tilde{I} \subset\{1,2 \ldots, k\}, \# \tilde{I}=k-m}{ }\left\|\sum_{i \in \tilde{I}}\right\| P_{A_{i}}(x)\left\|_{\ell} \cdot v_{i}\right\|_{V} .
$$

Then it follows that

$$
\gamma\left\|\sum_{i \in I}\right\| P_{A_{i}}(x)\left\|_{\ell} \cdot v_{i}\right\|_{V} \geq \frac{k-m}{k} \geq \frac{n-m}{n}
$$

Indeed

$$
\left(\begin{array}{c}
k \\
k-m
\end{array}\right) \cdot\left\|\sum_{i \in I}\right\| P_{A_{i}}(x)\left\|_{\ell} \cdot v_{i}\right\|_{V} \geq\left\|\sum_{\substack{\tilde{I} \subset\{1,2 \ldots, k\}, \# \tilde{I}=k-m}} \sum_{i \in \tilde{I}}\right\| P_{A_{i}}(x)\left\|_{\ell} \cdot v_{i}\right\|_{V}=\frac{1}{\gamma}\left(\begin{array}{c}
k-1 \\
k-m-1
\end{array}\right)
$$

and thus

$$
\gamma\left\|\sum_{i \in I}\right\| P_{A_{i}}(x)\left\|_{\ell} \cdot v_{i}\right\|_{V} \geq\left(\begin{array}{c}
k-1 \\
k-m-1
\end{array}\right) /\left(\begin{array}{c}
k \\
k-m
\end{array}\right)=\frac{k-m}{k} \geq \frac{n-m}{n} .
$$

Put

$$
\begin{aligned}
& I_{1}=\left\{i \in I: \# A_{i} \leq \min A_{i}-m\right\} \quad \text { and } \\
& I_{2}=I \backslash I_{1}=\left\{i \in I: \# A_{i}>\min A_{i}-m\right\}
\end{aligned}
$$

For $i \in I_{1}$ we have

$$
\left\|S^{m}\left(P_{A_{i}}(x)\right)\right\|_{\ell}=\left\|P_{A_{i}}(x)\right\|_{\ell} .
$$

Let $k^{\prime} \leq k-m$ and $i_{1}<i_{2}<\cdots<i_{k^{\prime}}$ be such that $I_{2}=\left\{i_{1}, i_{2}, \ldots, i_{k^{\prime}}\right\}$. We note that

$$
\begin{aligned}
& \min \left(A_{i_{1}}\right) \geq k \geq n, \\
& \min \left(A_{i_{2}}\right) \geq \min \left(A_{i_{1}}\right)+\# A_{i_{1}}>2 \cdot \min \left(A_{i_{1}}\right)-m \geq 2 n-m,
\end{aligned}
$$

and more generally, by induction, for each $s=2, \ldots, k^{\prime}$ we have

$$
\begin{aligned}
\min \left(A_{i_{s}}\right) & \geq \min \left(A_{i_{s-1}}\right)+\# A_{i_{s-1}}>2 \cdot \min \left(A_{i_{s-1}}\right)-m \\
& \geq 2 \cdot\left(2^{s-2} n-\left(2^{s-2}-1\right) m\right)-m=2^{s-1} n-\left(2^{s-1}-1\right) m .
\end{aligned}
$$


We deduce that

$$
\begin{aligned}
& \left\|S^{m}(x)\right\|_{\ell+1} \\
& \quad \geq \gamma\left\|\sum_{i \in I}\right\| S^{m}\left(P_{A_{i}}(x)\right)\left\|_{\ell} \cdot v_{i}\right\|_{V} \\
& \left.\quad \text { note that } \# I=k-m \leq \min A_{1}-m\right) \\
& \quad \geq \gamma\left\|\sum_{i \in I_{1}}\right\| P_{A_{i}}(x)\left\|_{\ell} \cdot v_{i}+\sum_{s=1}^{k^{\prime}}\left(1-\Delta\left(\ell, 2^{s-1} n-\left(2^{s-1}-1\right) m\right)\right)\right\| P_{A_{i_{s}}}(x)\left\|_{\ell} \cdot v_{i_{s}}\right\|_{V} \\
& \quad \text { (by the induction hypothesis and since }\left(v_{i}\right) \text { is } 1 \text {-unconditional) } \\
& \quad \geq \gamma\left\|\sum_{i \in I}\right\| P_{A_{i}}(x)\left\|_{\ell} \cdot v_{i}\right\|_{V}-\gamma \sum_{s=1}^{k^{\prime}} \Delta\left(\ell, 2^{s-1} n-\left(2^{s-1}-1\right) m\right) \\
& \quad\left(\text { since }\left\|P_{A_{i}}(x)\right\|_{\ell} \leq 1 \text { for } i=1,2, \ldots, k\right) \\
& \quad \geq \frac{n-m}{n}-\gamma \sum_{s=1}^{k^{\prime}} \sum_{t=0}^{\ell-1}(4 \gamma)^{t} \frac{m}{2^{s-1}(n-m)} \\
& \quad=1-\frac{m}{n}-\gamma \sum_{t=0}^{\ell-1}(4 \gamma)^{t} \frac{m}{(n-m)} \sum_{s=1}^{k^{\prime}} 2^{1-s} \\
& \quad \geq 1-\frac{m}{n-m}-4 \gamma \sum_{t=0}^{\ell-1}(4 \gamma)^{t} \frac{m}{(n-m)}=1-\Delta(\ell+1, n),
\end{aligned}
$$

which finishes the proof of our induction step.

Proposition 4.5. Let $V$ be a space with a normalized and 1-subsymmetric basis $\left(v_{i}\right)$. For a separable and reflexive Banach space $X$ the following are equivalent.

a) $X$ satisfies asymptotic $V$-lower tree estimates.

b) There exists $\gamma \in(0,1)$ such that $X$ satisfies $T(V, \gamma)$-lower tree estimates.

Proof. Using a result of Zippin [Z], and after renorming $X$ if necessary, we can assume that $X$ is (isometrically) a subspace of a reflexive space $Z$ with a bimonotone FDD $\left(E_{i}\right)$.

"(a) $\Rightarrow(\mathrm{b})$ " Let $C \geq 1$ so that $X$ satisfies asymptotic $C$ - $V$-lower tree estimates. For $k \in \mathbb{N}$ set

$$
\mathcal{A}^{(k)}=\left\{\left(x_{i}\right) \in S_{X}^{\omega}:\left\|\sum_{i=1}^{k} a_{i} v_{i}\right\|_{V} \leq C\left\|\sum_{i=1}^{k} a_{i} x_{i}\right\|_{X} \text { for all }\left(a_{i}\right)_{i=1}^{k} \subset \mathbb{R}\right\} .
$$

Let $\varepsilon>0$ be small enough so that for all $k \in \mathbb{N}$

$$
\overline{\mathcal{A}_{\varepsilon}^{(k)}} \subset\left\{\left(x_{i}\right) \in S_{X}^{\omega}:\left\|\sum_{i=1}^{k} a_{i} v_{i}\right\|_{V} \leq 2 C\left\|\sum_{i=1}^{k} a_{i} x_{i}\right\|_{X} \text { for all }\left(a_{i}\right)_{i=1}^{k} \subset \mathbb{R}\right\} .
$$

We let $\left(E_{i}^{(0)}\right)=\left(E_{i}\right)$ and apply Proposition 1.6 to $\mathcal{A}^{(k)}$ successively for each $k \in \mathbb{N}$ to obtain decreasing null sequences $\bar{\delta}^{(k)}=\left(\delta_{i}^{(k)}\right) \subset(0,1)$ and blockings $\left(E_{i}^{(k)}\right)$ of $\left(E_{i}^{(k-1)}\right)$ so that if $\left(x_{i}\right) \subset S_{X}$ is a $\bar{\delta}^{(k)}$-skipped block sequence of $\left(E_{i}^{(k)}\right)$, then $\left(x_{i}\right)$ lies in $\overline{\mathcal{A}_{\varepsilon}^{(k)}}$.

Let $\left(G_{i}\right)$ be a blocking of $\left(E_{i}\right)$ such that $\left(G_{i}\right)_{i=k}^{\infty}$ is a blocking of $\left(E_{i}^{(k)}\right)_{i=k}^{\infty}$ for all $k \in \mathbb{N}$. Then choose $\bar{\delta}=\left(\delta_{i}\right) \subset(0,1), \delta_{i} \downarrow 0$, such that if $\left(x_{i}\right) \subset S_{X}$ is a $\bar{\delta}$-skipped block sequence 
of $\left(G_{i}\right)$, then $\left(x_{i}\right)$ is a basic sequence with projection constant at most 2 and for any $k \in \mathbb{N}$ any normalized block sequence $\left(z_{i}\right)_{i=1}^{k}$ of $\left(x_{i}\right)_{i=k}^{\infty}$ is a $\bar{\delta}^{(k)}$-skipped block sequence of $\left(E_{i}^{(k)}\right)$. It follows that any $\bar{\delta}$-skipped block sequence of $\left(G_{i}\right)$ is a basic sequence with projection constant at most 2 satisfying asymptotic $(2 C)-V$-lower estimates, and hence, by Proposition 4.2, it 2-dominates the unit vector basis $\left(t_{i}\right)$ of $T(V, \gamma)$ for $\gamma=(4 C)^{-1}$. Thus (b) follows.

"(b) $\Rightarrow$ (a)" This follows from Proposition 1.1 and the fact that, by (39), $\left(t_{i}\right)_{i=k}^{2 k-1} C$-dominates $\left(v_{i}\right)_{i=1}^{k}$ with $C=\gamma^{-1}$, where $\left(t_{i}\right)$ is the unit vector basis of $T(V, \gamma)$.

We are now ready to state the main results of this section.

Theorem 4.6. Assume that $V$ is a space with a normalized and 1-subsymmetric basis $\left(v_{i}\right)$ and that $X$ is a separable, reflexive space with asymptotic $V$-lower tree estimates. Then $X$ can be embedded in a reflexive space $Z$ with an FDD satisfying asymptotic $V$-lower estimates, and $X$ is isomorphic to a quotient of a reflexive space $Y$ with an FDD satisfying asymptotic $V$-lower estimates.

Proof. If $\left(v_{i}\right)$ is equivalent to the unit vector basis of $c_{0}$, then we simply use the theorem of Zippin [Z to embed $X$ and $X^{*}$ into reflexive spaces $Z$ and $Y^{*}$, respectively, with FDDs. The result then follows, since any FDD satisfies (asymptotic) $c_{0}$-lower estimates.

Assume now that $\left(v_{i}\right)$ is not equivalent to the unit vector basis of $c_{0}$. By Proposition 4.5 $X$ satisfies $T(V, \gamma)$-lower tree estimates for some $\gamma \in(0,1)$. We may clearly assume that $\gamma<1 / 4$, and then, by Proposition 4.3. $T(V, \gamma)$ is a reflexive space whose unit vector basis $\left(t_{i}\right)$ is a normalized and 1-unconditional basis satisfying $(S R S)$ and $(W L S)$ and dominated by its normalized block sequences. Hence, by Corollary [3.2 $X$ embeds into a reflexive space $Z$ with an FDD satisfying $T(V, \gamma)$-lower estimates, and $X$ is isomorphic to a quotient of a reflexive space $Y$ with an FDD satisfying $T(V, \gamma)$-lower estimates. It is clear (e.g. from (39)) that the FDDs of $Z$ and $Y$ satisfy asymptotic $V$-lower esimates.

Theorem 4.7. Let $V$ and $U$ be Banach spaces with normalized, 1-subsymmetric bases $\left(v_{i}\right)$ and $\left(u_{i}\right)$, respectively. Assume that, for any $\gamma \in(0,1 / 4)$, every normalized block sequence of the unit vector basis of $\left.\left(T\left(U^{*}\right), \gamma\right)\right)^{*}$ dominates every normalized block sequence of the unit vector basis of $T(V, \gamma)$. For a separable and reflexive Banach space $X$ the following are equivalent.

a) $X$ satisfies asymptotic $(V, U)$-tree estimates.

b) $X$ can be embedded in a reflexive space $Z$ with an $F D D\left(E_{i}\right)$ satisfying asymptotic $(V, U)$-estimates.

c) $X$ is the quotient of a reflexive space $Z$ with an $F D D\left(E_{i}\right)$ satisfying asymptotic $(V, U)$-estimates.

d) $X^{*}$ satisfies asymptotic $\left(U^{(*)}, V^{(*)}\right)$-tree estimates.

Remark. The conditions of the theorem are satisfied by certain pairs of $\ell_{p}$ spaces. This will be spelt out in Corollary 4.8 below. Note that $T\left(\mathrm{c}_{0}, \gamma\right)$ is just the space $\mathrm{c}_{0}$ for any $\gamma \in(0,1)$. So if one of the bases $\left(v_{i}\right)$ and $\left(u_{i}^{*}\right)$ of $V$ and $U^{(*)}$, respectively, are equivalent to the unit vector basis of $c_{0}$, then the assumptions on the spaces $T(V, \gamma)$ and $\left(T\left(U^{(*)}, \gamma\right)\right)^{*}$ are automatically satisfied. In this case the theorem is really a statement about one-sided estimates since every reflexive space satisfies $\left(c_{0}, \ell_{1}\right)$-tree estimates, and any FDD satisfies $\left(c_{0}, \ell_{1}\right)$-estimates. 
Proof. First note that the implication "(b) $\Rightarrow(\mathrm{a})$ " is clear and, by Proposition 4.1 (a), that the implication " $(\mathrm{c}) \Rightarrow(\mathrm{d})$ " is just another instance of "(b) $\Rightarrow(\mathrm{a})$ ". Also, the implications "(a) $\Rightarrow(\mathrm{d})$ " and "(d) $\Rightarrow(\mathrm{a})$ " are equivalent since the pair $\left(U^{(*)}, V^{(*)}\right)$ satisfies the same assumptions as the pair $(V, U)$. Thus we will have completed the proof once we show how to deduce (b) and (c) from (a).

The assumption that (a) holds splits into the following two conditions by Proposition 4.1 (b): $X$ satisfies asymptotic $V$-lower tree estimates, and $X^{*}$ satisfies asymptotic $U^{(*)}$-lower tree estimates. If $\left(u_{i}^{*}\right)$ is equivalent to the unit vector basis of $c_{0}$, then the second condition is redundant (cf. the remark preceding this proof), and (b) and (c) follow from an application of Theorem [4.6 to the pair $(V, X)$. Similarly, if $\left(v_{i}\right)$ is equivalent to the unit vector basis of $c_{0}$, then the first condition is redundant, and (b) and (c) follow from an application of Theorem 4.6 to the pair $\left(U^{(*)}, X^{*}\right)$ followed by an application of Theorem 4.1 (a). In general, by Proposition 4.5 it follows from the two conditions that, for some $\gamma \in(0,1 / 4), X$ satisfies $T(V, \gamma)$-lower tree estimates, and $X^{*}$ satisfies $T\left(U^{(*)}, \gamma\right)$-lower tree estimates.

We now continue the proof under the assumption that neither $\left(v_{i}\right)$ nor $\left(u_{i}^{*}\right)$ is equivalent to the unit vector basis of $c_{0}$. Then it follows from Proposition 4.3 that $T(V, \gamma)$ and $T\left(U^{(*)}, \gamma\right)$ are reflexive spaces each having a normalized, 1-unconditional basis satisfying $(S R S)$ and $(W L S)$ and dominated by all its normalized block sequences. Thus we are in the situation of Corollary 3.3. two applications of which give that $X$ satisfies $\left(T(V, \gamma), T\left(U^{(*)}, \gamma\right)^{*}\right)$-tree estimates, and $X^{*}$ satisfies $\left(T\left(U^{(*)}, \gamma\right), T(V, \gamma)^{*}\right)$-tree estimates.

We now complete the proof by applying Theorem 3.4 first to $\left(T(V, \gamma), T\left(U^{(*)}, \gamma\right)^{*}\right)$ and $X$ to deduce (b) and then to $\left(T\left(U^{(*)}, \gamma\right), T(V, \gamma)^{*}\right)$ and $X^{*}$ to obtain (c) (the second application of Theorem 3.4 is followed by an application of Proposition 4.1 (a)). Note that we are assuming that every normalized block sequence of the unit vector basis of $\left(T\left(U^{(*)}, \gamma\right)\right)^{*}$ dominates every normalized block sequence of the unit vector basis of $T(V, \gamma)$ (which then implies the same for the spaces $T(V, \gamma)^{*}$ and $\left.\left(T\left(U^{*}\right), \gamma\right)\right)$, so the conditions of Theorem 3.4 are indeed satisfied.

Let $0<\gamma<1$ and $1 \leq p<\infty$. We shall write $T_{p, \gamma}$ for the Tsirelson space $T\left(\ell_{p}, \gamma\right)$ associated to $\ell_{p}$ and $\gamma$.

Corollary 4.8. Let $1 \leq q \leq p \leq \infty$, and let $\frac{1}{p}+\frac{1}{p^{\prime}}=1$ and $\frac{1}{q}+\frac{1}{q^{\prime}}=1$. For a separable and reflexive Banach space $X$ the following are equivalent.

a) $X$ satisfies asymptotic $\left(\ell_{p}, \ell_{q}\right)$-tree estimates.

b) $X$ can be embedded in a reflexive space $Z$ with an FDD $\left(E_{i}\right)$ satisfying asymptotic $\left(\ell_{p}, \ell_{q}\right)$-estimates.

c) $X$ is the quotient of a reflexive space $Z$ with an $F D D\left(E_{i}\right)$ satisfying asymptotic $\left(\ell_{p}, \ell_{q}\right)$-estimates.

d) $X^{*}$ satisfies asymptotic $\left(\ell_{q^{\prime}}, \ell_{p^{\prime}}\right)$-tree estimates.

Remark. Following usual custom the range $\ell_{p}, 1 \leq p \leq \infty$ really means the range $\ell_{p}$, $1 \leq p<\infty, \mathrm{c}_{0}$.

Proof. We will verify that the conditions of Theorem 4.7 hold for $V=\ell_{p}$ and $U=\ell_{q}$. By the remark following Theorem 4.7 these conditions are automatically satisfied if $p=\infty$ or $q=1$. Let us now assume that $1<q \leq p<\infty$. 
Since $T_{p, \gamma}$ is the $p$-convexification of $T_{1, \gamma^{p}}$ (see [CS]), the unit vector basis of $T_{p, \gamma}$ is 1-dominated by the unit vector basis of $\ell_{p}$, and hence the unit vector basis of $\left(T_{p, \gamma}\right)^{*} 1$ dominates the unit vector basis of $\ell_{p^{\prime}}$. From this one can easile deduce that every normalized block sequence of the unit vector basis of $\left(T_{q^{\prime}, \gamma}\right)^{*}$ 1-dominates every normalized block sequence of the unit vector basis of $T_{p, \gamma}$.

A special case of Corollary 4.8 solves Problem 5.4 raised in OS1].

Corollary 4.9. Let $X$ be a reflexive asymptotic $\ell_{p}$ space (meaning that $X$ satisfies asymptotic $\left(\ell_{p}, \ell_{p}\right)$-tree estimates) for some $p$ with $1 \leq p \leq \infty$. Then $X$ can be embedded into a reflexive space with an asymptotic $\ell_{p} F D D$, and $X$ is the quotient of a reflexive space with an asymptotic $\ell_{p} F D D$.

\section{REFERENCES}

[B] J. Bourgain, On separable Banach spaces, universal for all separable reflexive spaces, Proc. Amer. Math. Soc. 79 (1980), no. 2, 241-246.

[CS] P. G. Casazza and T. J. Shura Tsirelson's Space, Lecture Notes in Mathematics 1363, SpringerVerlag (1989).

[FJ] Figiel and W. B. Johnson, A uniformly convex Banach space which contains no $\ell_{p}$, Comp. Math. 29 (1974), 179-190

[J] W.B. Johnson, on quotients of $L_{p}$ which are quotients of $\ell_{p}$, Compositio Math. 34 (1977), $69-89$.

[JZ1] W.B. Johnson and M. Zippin, On subspaces of quotients of $\left(\sum G_{n}\right)_{l_{p}}$ and $\left(\sum G_{n}\right)_{c_{0}}$, Proceedings of the International Symposium on Partial Differential Equations and the Geometry of Normed Linear Spaces, Jerusalem, 1972, Israel J. Math. 13 (1972), 311-316 (1973).

[JZ2] W.B. Johnson and M. Zippin, Subspaces and quotient spaces of $\left(\sum G_{n}\right)_{\ell_{p}}$ and $\left(\sum G_{n}\right)_{c_{0}}$, Israel J. Math. 17 (1974), 50-55.

[KOS] H. Knaust, E. Odell, and Th. Schlumprecht, On asymptotic structure, the Szlenk index and UKK properties in Banach spaces, Positivity 3 (1999), 173-199.

[LT] J. Lindenstrauss and L. Tzafriri, Classical Banach Spaces, I, Springer-Verlag New York (1977).

[MMT] B. Maurey, V.D. Milman and N. Tomczak-Jaegermann, Asymptotic infinite-dimensional theory of Banach spaces, Oper. Theory: Adv. Appl. 77 (1994), 149-175.

[MT] V.D. Milman and N. Tomczak-Jaegermann, Asymptotic $\ell_{p}$ spaces and bounded distortions, eds. Bor-Luh Lin and W.B. Johnson, Contemp. Math. 144 (1993), 173-195.

[OS1] E. Odell, and Th. Schlumprecht, Trees and branches in Banach spaces, Trans. Amer. Math. Soc. 354 (2002), no. 10, 4085-4108.

[OS2] E. Odell, and Th. Schlumprecht, A universal reflexive space for the class of uniformly convex Banach spaces, to appear in the Mathematische Annalen.

$[\mathrm{P}] \quad$ S. Prus, Finite-dimensional decompositions of Banach spaces with $(p, q)$-estimates, Dissertationes Math. 263 (1987).

[Z] M. Zippin, Banach spaces with separable duals, Trans. AMS, 310, Nr. 1 (1988), 371-379. 